\title{
Positive Solutions and Mann Iterative Algorithms for a Second-Order Nonlinear Difference Equation
}

\author{
Zeqing Liu, ${ }^{1}$ Xin $\mathrm{Li}^{2}{ }^{2}$ Shin Min Kang, ${ }^{3}$ and Young Chel Kwun ${ }^{4}$ \\ ${ }^{1}$ Department of Mathematics, Liaoning Normal University, Dalian, Liaoning 116029, China \\ ${ }^{2}$ Suizhong No. 1 Senior High School, Huludao, Liaoning 125200, China \\ ${ }^{3}$ Department of Mathematics and RINS, Gyeongsang National University, Jinju 52828, Republic of Korea \\ ${ }^{4}$ Department of Mathematics, Dong-A University, Busan 49315, Republic of Korea
}

Correspondence should be addressed to Young Chel Kwun; yckwun@dau.ac.kr

Received 17 December 2015; Accepted 24 February 2016

Academic Editor: Jozef Banas

Copyright (C) 2016 Zeqing Liu et al. This is an open access article distributed under the Creative Commons Attribution License, which permits unrestricted use, distribution, and reproduction in any medium, provided the original work is properly cited.

\begin{abstract}
This paper deals with the second-order nonlinear neutral delay difference equation $\Delta\left(a_{n} h\left(x_{n-\tau_{1 n}}, x_{n-\tau_{2 n}}, \ldots, x_{n-\tau_{m n}}\right) \Delta\left(x_{n}-q_{n} x_{n-\tau_{0}}\right)\right)+$ $f\left(n, x_{n-\sigma_{1 n}}, x_{n-\sigma_{2 n}}, \ldots, x_{n-\sigma_{k n}}\right)=b_{n}, n \geq n_{0}$. Using the Banach fixed point theorem, Mann iterative method with errors, and some new techniques, we prove the existence of uncountably many positive solutions and the convergence of the sequences generated by the Mann iterative method with errors relative to these solutions for the above equation. Six examples are included. Our results extend and improve essentially the known results in this field.
\end{abstract}

\section{Introduction and Preliminaries}

Consider the second-order nonlinear neutral delay difference equation with forcing term:

$$
\begin{aligned}
& \Delta\left(a_{n} h\left(x_{n-\tau_{1 n}}, x_{n-\tau_{2 n}}, \ldots, x_{n-\tau_{m n}}\right) \Delta\left(x_{n}-q_{n} x_{n-\tau_{0}}\right)\right) \\
& \quad+f\left(n, x_{n-\sigma_{1 n}}, x_{n-\sigma_{2 n}}, \ldots, x_{n-\sigma_{k n}}\right)=b_{n}, \quad n \geq n_{0},
\end{aligned}
$$

where $n_{0} \in \mathbb{N}_{0}, m, k, \tau_{0} \in \mathbb{N}, \bigcup_{i \in\{1,2, \ldots, m\}}\left\{\tau_{i n}\right\}_{n \in \mathbb{N}_{n_{0}}} \subset \mathbb{Z}$, $\bigcup_{j \in\{1,2, \ldots, k\}}\left\{\sigma_{j n}\right\}_{n \in \mathbb{N}_{n_{0}}} \subset \mathbb{Z}$ with

$$
\begin{aligned}
\lim _{n \rightarrow \infty}\left(n-\tau_{\text {in }}\right)=\lim _{n \rightarrow \infty}\left(n-\sigma_{j n}\right)=+\infty, \\
\quad i \in\{1,2, \ldots, m\}, j \in\{1,2, \ldots, k\},
\end{aligned}
$$

$\left\{a_{n}\right\}_{n \in \mathbb{N}_{n_{0}}},\left\{b_{n}\right\}_{n \in \mathbb{N}_{n_{0}}}$, and $\left\{q_{n}\right\}_{n \in \mathbb{N}_{n_{0}}}$ are real sequences with $a_{n} \neq$ 0 for each $n \in \mathbb{N}_{n_{0}}, f \in C\left(\mathbb{N}_{n_{0}} \times \mathbb{R}^{k}, \mathbb{R}\right)$, and $h \in C\left(\mathbb{R}^{m}, \mathbb{R}\right)$.

In the recent ten years, there have been a lot of activities concerning the oscillation, nonoscillation, asymptotic behavior, and existence of solutions, nonoscillatory solutions, and positive solutions for various types of difference equations, which are special cases of (1); see, for example, [1-21] and the references cited therein. In 2007, Cheng [3] used the Banach fixed point theorem to investigate the second-order neutral delay linear difference equation with positive and negative coefficients:

$$
\Delta^{2}\left(x_{n}+p x_{n-m}\right)+p_{n} x_{n-k}-q_{n} x_{n-l}=0, \quad n \geq n_{0},
$$

and got a sufficient condition for the existence of a nonoscillatory solution of (3). In 2004, Malaguti et al. [15] discussed the second-order nonlinear difference equation

$$
\Delta\left(r_{k} \Delta u_{k}\right)+q_{k} g\left(u_{k+1}\right)=0, \quad k \geq 0,
$$

and obtained sufficient and necessary conditions for the existence of bounded solutions of (4). In 2007, by using the Krasnoselskii fixed point theorem and Schauder fixed point theorem, Rath et al. [16] investigated the existence of solutions of the second-order nonlinear neutral delay difference equation

$$
\Delta\left(r_{n} \Delta\left(x_{n}-p_{n} x_{n-m}\right)\right)+q_{n} G\left(x_{n-k}\right)=0, \quad n \geq n_{0} .
$$


In 2003, Agarwal et al. [1] used a nonlinear alternative of Leray-Schauder type to obtain the existence of a nonoscillatory solution for the following second-order neutral delay difference equation:

$$
\Delta\left(a_{n} \Delta\left(y_{n}+p y_{n-\tau}\right)\right)+F\left(n+1, y_{n+1-\sigma}\right)=0, \quad n \geq 1
$$

In 2009, using the Banach fixed point theorem, Liu et al. [12] researched the global solvability for the second-order nonlinear neutral delay difference equation

$$
\begin{aligned}
& \Delta\left(a_{n} \Delta\left(x_{n}+b x_{n-\tau}\right)\right)+f\left(n, x_{n-d_{1 n}}, x_{n-d_{2 n}}, \ldots, x_{n-d_{d n}}\right) \\
& \quad=c_{n}, \quad n \geq n_{0} .
\end{aligned}
$$

However, the study of the existence of bounded positive solutions for a lot of second-order neutral delay difference equations appears to be insufficient. In particular, the uncountably many bounded positive solutions for these second-order neutral delay difference equations obtain less concern.

The purpose of this paper is to study the solvability and convergence of Mann iterative approximations of (1). By means of the Banach fixed point theorem, Mann iterative method with errors, and a few new techniques, we obtain the existence of uncountably many bounded positive solutions of (1) and prove that the sequences generated by the Mann iterative method with errors converge to these bounded positive solutions. Our results generalize and improve all results in $[3,12]$. Six examples are constructed in order to illustrate the superiority and applications of the results presented in this paper.

Throughout this paper, we assume that $\mathbb{R}=(-\infty,+\infty)$, $\mathbb{N}_{0}=\{0\} \cup \mathbb{N}, \mathbb{N}$ and $\mathbb{Z}$ denote the sets of all positive integers and integers, respectively, and $\Delta$ stands for the forward difference operator; that is, $\Delta x_{n}=x_{n+1}-x_{n}$. Let

$$
\begin{aligned}
& \beta=\inf \left\{n-\tau_{0}, n-\tau_{i n}, n-\sigma_{j n}: n \in \mathbb{N}_{n_{0}}, 1 \leq i \leq k, 1\right. \\
& \leq j \leq m\} \text {, } \\
& \mathbb{Z}_{\beta}=\{n: n \in \mathbb{Z} \text { with } n \geq \beta\}, \\
& \mathbb{N}_{t}=\left\{n: n \in \mathbb{N}_{0} \text { with } n \geq t\right\}, \quad \forall t \in \mathbb{N}_{0} .
\end{aligned}
$$

Assume that $l_{\beta}^{\infty}$ represents the Banach space of all bounded real sequences $x=\left\{x_{n}\right\}_{n \in \mathbb{Z}_{\beta}}$ with the norm $\|x\|=\sup _{n \in \mathbb{Z}_{\beta}}\left|x_{n}\right|$ and

$$
\begin{array}{r}
A(N, M)=\left\{x=\left\{x_{n}\right\} \in l_{\beta}^{\infty}: N \leq x_{n} \leq M, n \in \mathbb{Z}_{\beta}\right\} \\
\forall M>N>0 .
\end{array}
$$

It is easy to see that $A(N, M)$ is a bounded closed and convex subset of the Banach space $l_{\beta}^{\infty}$.

By a solution of (1), we mean a real sequence $\left\{x_{n}\right\}_{n \in \mathbb{Z}_{\beta}}$ with a positive integer $D \geq n_{0}+\tau_{0}+|\beta|$ such that (1) holds for all $n \geq D$.
Lemma 1 (see [22]). Let $\left\{\alpha_{n}\right\}_{n \geq 0},\left\{\beta_{n}\right\}_{n \geq 0},\left\{\gamma_{n}\right\}_{n \geq 0}$, and $\left\{t_{n}\right\}_{n \geq 0}$ be four nonnegative real sequences satisfying the inequality

$$
\alpha_{n+1} \leq\left(1-t_{n}\right) \alpha_{n}+t_{n} \beta_{n}+\gamma_{n}, \quad \forall n \geq 0,
$$

where $\left\{t_{n}\right\}_{n \geq 0} \subseteq[0,1], \sum_{n=0}^{\infty} t_{n}=+\infty, \lim _{n \rightarrow \infty} \beta_{n}=0$, and $\sum_{n=0}^{\infty} \gamma_{n}<+\infty$. Then, $\lim _{n \rightarrow \infty} \alpha_{n}=0$.

Lemma 2. Let $\tau \in \mathbb{N}, n_{0} \in \mathbb{N}_{0}$ be two constants and let $\left\{c_{n}\right\}_{n \in \mathbb{N}_{n_{0}+1}},\left\{k_{n}\right\}_{n \in \mathbb{N}_{n_{0}}}$ be two nonnegative sequences. Then,

$$
\begin{aligned}
& \sum_{j=0}^{\infty} \sum_{i=n_{0}+1+j \tau}^{\infty}\left(c_{i} \sum_{s=n_{0}}^{i-1} k_{s}\right)<+\infty \Longleftrightarrow \sum_{i=n_{0}+1}^{\infty}\left(i c_{i} \sum_{s=n_{0}}^{i-1} k_{s}\right) \\
& \quad<+\infty .
\end{aligned}
$$

Proof. For each $t \in \mathbb{R},[t]$ denotes the largest integer not exceeding $t$. Notice that

$$
\begin{aligned}
& \sum_{j=0}^{\infty} \sum_{i=n_{0}+1+j \tau}^{\infty}\left(c_{i} \sum_{s=n_{0}}^{i-1} k_{s}\right) \\
&= \sum_{i=n_{0}+1}^{\infty}\left(c_{i} \sum_{s=n_{0}}^{i-1} k_{s}\right)+\sum_{i=n_{0}+1+\tau}^{\infty}\left(c_{i} \sum_{s=n_{0}}^{i-1} k_{s}\right) \\
&+\sum_{i=n_{0}+1+2 \tau}^{\infty}\left(c_{i} \sum_{s=n_{0}}^{i-1} k_{s}\right)+\cdots \\
&= \sum_{i=n_{0}+1}^{\infty}\left\{1+\left[\frac{i-n_{0}-1}{\tau}\right]\right\}\left(c_{i} \sum_{s=n_{0}}^{i-1} k_{s}\right), \\
& \lim _{i \rightarrow \infty} \frac{1+\left[\left(i-n_{0}-1\right) / \tau\right]}{i / \tau}=1 .
\end{aligned}
$$

Thus, (11) follows from (12). This completes the proof.

\section{Main Results}

According to the different ranges of the sequence $\left\{q_{n}\right\}_{n \in \mathbb{N}_{n_{0}}}$, now we use the Banach fixed point theorem to study the existence of uncountably many bounded positive solutions and convergence of the Mann iterative approximations of (1).

Theorem 3. Assume that there exist constants $M, N, q$, $L_{h}$, and $m_{h}$ and two nonnegative sequences $\left\{P_{n}\right\}_{n \in \mathbb{N}_{n_{0}}}$ and $\left\{W_{n}\right\}_{n \in \mathbb{N}_{n_{0}}}$ satisfying

$$
\begin{aligned}
& 0<N<(1-q) M \\
& 0 \leq q_{n} \leq q<1, \text { eventually; }
\end{aligned}
$$




$$
\begin{aligned}
& \left|h\left(u_{1}, u_{2}, \ldots, u_{m}\right)-h\left(\bar{u}_{1}, \bar{u}_{2}, \ldots, \bar{u}_{m}\right)\right| \\
& \leq L_{h} \max \left\{\left|u_{i}-\bar{u}_{i}\right|: 1 \leq i \leq m\right\}, \\
& \forall\left(u_{i}, \bar{u}_{i}\right) \in[N, M]^{2}, 1 \leq i \leq m ; \\
& \left|h\left(u_{1}, u_{2}, \ldots, u_{m}\right)\right| \geq m_{h}>0, \\
& \forall u_{i} \in[N, M], 1 \leq i \leq m ; \\
& \left|f\left(n, u_{1}, u_{2}, \ldots, u_{k}\right)-f\left(n, \bar{u}_{1}, \bar{u}_{2}, \ldots, \bar{u}_{k}\right)\right| \\
& \leq P_{n} \max \left\{\left|u_{i}-\bar{u}_{i}\right|: 1 \leq i \leq m\right\}, \\
& \forall\left(n, u_{i}, \bar{u}_{i}\right) \in \mathbb{N}_{n_{0}} \times[N, M]^{2}, 1 \leq i \leq m ;
\end{aligned}
$$

$$
\begin{gathered}
\left|f\left(n, u_{1}, u_{2}, \ldots, u_{k}\right)\right| \leq W_{n}, \\
\forall\left(n, u_{i}\right) \in \mathbb{N}_{n_{0}} \times[N, M], 1 \leq i \leq m ; \\
\sum_{i=n_{0}+1}^{\infty} \frac{1}{\left|a_{i}\right|} \sum_{s=n_{0}}^{i-1} \max \left\{W_{s}, P_{s},\left|b_{s}\right|\right\}<+\infty .
\end{gathered}
$$

Then, one has the following.

(a) For any $c \in(N,(1-q) M)$, there exist $\theta \in(0,1)$ and $D \geq 1+n_{0}+\tau_{0}+|\beta|$ such that, for each $x_{0}=\left\{x_{0, n}\right\}_{n \in \mathbb{Z}_{\beta}} \in$ $A(N, M)$, the Mann iterative sequence with errors $\left\{x_{\eta}\right\}_{\eta \in \mathbb{N}_{0}}=$ $\left\{x_{\eta, n}\right\}_{(\eta, n) \in \mathbb{N}_{0} \times \mathbb{Z}_{\beta}}$ generated by the scheme

$$
\begin{aligned}
& x_{\eta+1, n} \\
& =\left\{\begin{array}{l}
\left(1-\alpha_{\eta}-\beta_{\eta}\right) x_{\eta, n}+\alpha_{\eta}\left\{c+q_{n} x_{\eta, n-\tau_{0}}+\sum_{i=n}^{\infty} \frac{1}{a_{i} h\left(x_{\eta, i-\tau_{1 i}}, x_{\eta, i-\tau_{2 i}}, \ldots, x_{\eta, i-\tau_{m i}}\right)} \sum_{s=n_{0}}^{i-1}\left[f\left(s, x_{\eta, s-\sigma_{1 s}}, x_{\eta, s-\sigma_{2 s}}, \ldots, x_{\eta, s-\sigma_{k s}}\right)-b_{s}\right]\right\}+\beta_{\eta} \gamma_{\eta, n}, \quad n \geq D, \eta \geq 0, \\
\left(1-\alpha_{\eta}-\beta_{\eta}\right) x_{\eta, D}+\alpha_{\eta}\left\{c+q_{D} x_{\eta, D-\tau_{0}}+\sum_{i=D}^{\infty} \frac{1}{a_{i} h\left(x_{\eta, i-\tau_{1 i}}, x_{\eta, i-\tau_{2 i}}, \ldots, x_{\eta, i-\tau_{m i}}\right)} \sum_{s=n_{0}}^{i-1}\left[f\left(s, x_{\eta, s-\sigma_{1 s}}, x_{\eta, s-\sigma_{2 s}}, \ldots, x_{\eta, s-\sigma_{k s}}\right)-b_{s}\right]\right\}+\beta_{\eta} \gamma_{\eta, D}, \quad \beta \leq n<D, \eta \geq 0,
\end{array}\right.
\end{aligned}
$$

converges to a bounded positive solution $x \in A(N, M)$ of (1) and has the following error estimate:

$$
\begin{aligned}
& \left\|x_{\eta+1}-x\right\| \leq\left(1-(1-\theta) \alpha_{\eta}\right)\left\|x_{\eta}-x\right\|+2 M \beta_{\eta}, \\
& \eta \geq 0,
\end{aligned}
$$

where $\left\{\gamma_{\eta}\right\}_{\eta \in \mathbb{N}_{0}}=\left\{\gamma_{\eta, n}\right\}_{(\eta, n) \in \mathbb{N}_{0} \times \mathbb{Z}_{\beta}}$ is an arbitrary sequence in $A(N, M)$ and $\left\{\alpha_{\eta}\right\}_{\eta \in \mathbb{N}_{0}}$ and $\left\{\beta_{\eta}\right\}_{\eta \in \mathbb{N}_{0}}$ are any sequences in $[0,1]$ such that

$$
\begin{aligned}
& \sum_{\eta=0}^{\infty} \alpha_{\eta}=+\infty, \\
& \sum_{\eta=0}^{\infty} \beta_{\eta}<+\infty
\end{aligned}
$$

or there exists a sequence $\left\{\xi_{\eta}\right\}_{\eta \geq 0} \subset[0,+\infty)$ satisfying $\beta_{\eta}=\xi_{\eta} \alpha_{\eta}, \forall \eta \in \mathbb{N}_{0}, \lim _{\eta \rightarrow \infty} \xi_{\eta}=0$. (b) Equation (1) has uncountably many bounded positive solutions in $A(N, M)$.

Proof. First, we show that (a) holds. Let $c \in(N,(1-q) M)$. It follows from (14) and (19) that there exist $\theta \in(0,1)$ and $D \geq 1+n_{0}+\tau_{0}+|\beta|$ sufficiently large such that

$$
\begin{aligned}
& 0 \leq q_{n} \leq q<1, \quad \forall n \geq D, \\
& \theta=q+\frac{1}{m_{h}^{2}} \sum_{i=D}^{\infty} \frac{1}{\left|a_{i}\right|} \sum_{s=n_{0}}^{i-1}\left[m_{h} P_{s}+L_{h}\left(W_{s}+\left|b_{s}\right|\right)\right], \\
& \frac{1}{m_{h}} \sum_{i=D}^{\infty} \frac{1}{\left|a_{i}\right|} \sum_{s=n_{0}}^{i-1}\left(W_{s}+\left|b_{s}\right|\right) \\
& \quad<\min \{c-N,(1-q) M-c\} .
\end{aligned}
$$

Define an operator $T: A(N, M) \rightarrow l_{\beta}^{\infty}$ by

$$
T x_{n}= \begin{cases}c+q_{n} x_{n-\tau_{0}}+\sum_{i=n}^{\infty} \frac{1}{a_{i} h\left(x_{i-\tau_{1 i}}, x_{i-\tau_{2 i}}, \ldots, x_{i-\tau_{m i}}\right)} \sum_{s=n_{0}}^{i-1}\left[f\left(s, x_{s-\sigma_{1 s}}, x_{s-\sigma_{2 s}}, \ldots, x_{s-\sigma_{k s}}\right)-b_{s}\right], & n \geq D, \\ T x_{D}, & \beta \leq n<D,\end{cases}
$$

for any $x=\left\{x_{n}\right\}_{n \in \mathbb{Z}_{\beta}} \in A(N, M)$. On account of (15) (18) and (23) (24), we get that, for every $x=\left\{x_{n}\right\}_{n \in \mathbb{Z}_{\beta}}, y=\left\{y_{n}\right\}_{n \in \mathbb{Z}_{\beta}} \in$ $A(N, M)$, and $n \geq D$,

$$
\begin{gathered}
T x_{n}=c+q_{n} x_{n-\tau_{0}}+\sum_{i=n}^{\infty} \frac{1}{a_{i} h\left(x_{i-\tau_{1 i}}, x_{i-\tau_{2 i}}, \ldots, x_{i-\tau_{m i}}\right)} \\
\cdot \sum_{s=n_{0}}^{i-1}\left[f\left(s, x_{s-\sigma_{1 s}}, x_{s-\sigma_{2 s}}, \ldots, x_{s-\sigma_{k s}}\right)-b_{s}\right] \geq c
\end{gathered}
$$

$$
\begin{aligned}
& -\frac{1}{m_{h}} \sum_{i=n}^{\infty} \frac{1}{\left|a_{i}\right|} \sum_{s=n_{0}}^{i-1}\left[\left|f\left(s, x_{s-\sigma_{1 s}}, x_{s-\sigma_{2 s}}, \ldots, x_{s-\sigma_{k s}}\right)\right|\right. \\
& \left.+\left|b_{s}\right|\right] \geq c-\frac{1}{m_{h}} \sum_{i=n}^{\infty} \frac{1}{\left|a_{i}\right|} \sum_{s=n_{0}}^{i-1}\left(W_{s}+\left|b_{s}\right|\right) \geq c-\min \{c \\
& -N,(1-q) M-c\} \geq N, \\
& T x_{n}=c+q_{n} x_{n-\tau_{0}}+\sum_{i=n}^{\infty} \frac{1}{a_{i} h\left(x_{i-\tau_{1 i}}, x_{i-\tau_{2 i}}, \ldots, x_{i-\tau_{m i}}\right)}
\end{aligned}
$$




$$
\begin{aligned}
& \cdot \sum_{s=n_{0}}^{i-1}\left[f\left(s, x_{s-\sigma_{1 s}}, x_{s-\sigma_{2 s}}, \ldots, x_{s-\sigma_{k s}}\right)-b_{s}\right] \leq c \\
& +q M+\frac{1}{m_{h}} \sum_{i=n}^{\infty} \frac{1}{\left|a_{i}\right|} \\
& \cdot \sum_{s=n_{0}}^{i-1}\left[\left|f\left(s, x_{s-\sigma_{1 s}}, x_{s-\sigma_{2 s}}, \ldots, x_{s-\sigma_{k s}}\right)\right|+\left|b_{s}\right|\right] \leq c \\
& +q M+\frac{1}{m_{h}} \sum_{i=n}^{\infty} \frac{1}{\left|a_{i}\right|} \sum_{s=n_{0}}^{i-1}\left(W_{s}+\left|b_{s}\right|\right) \leq c+q M \\
& +\min \{c-N,(1-q) M-c\} \leq M, \\
& \left|T x_{n}-T y_{n}\right|=\mid q_{n}\left(x_{n-\tau_{0}}-y_{n-\tau_{0}}\right) \\
& +\sum_{i=n}^{\infty} \frac{1}{a_{i} h\left(x_{i-\tau_{1 i}}, x_{i-\tau_{2 i}}, \ldots, x_{i-\tau_{m i}}\right)} \\
& \cdot \sum_{s=n_{0}}^{i-1}\left[f\left(s, x_{s-\sigma_{1 s}}, x_{s-\sigma_{2 s}}, \ldots, x_{s-\sigma_{k s}}\right)-b_{s}\right] \\
& -\sum_{i=n}^{\infty} \frac{1}{a_{i} h\left(y_{i-\tau_{1 i}}, y_{i-\tau_{2 i}}, \ldots, y_{i-\tau_{m i}}\right)} \\
& \cdot \sum_{s=n_{0}}^{i-1}\left[f\left(s, y_{s-\sigma_{1 s}}, y_{s-\sigma_{2 s}}, \ldots, y_{s-\sigma_{k s}}\right)-b_{s}\right] \mid \leq q \| x \\
& -y \|+\sum_{i=n}^{\infty} \frac{1}{\left|a_{i}\right|} \sum_{s=n_{0}}^{i-1}\left[\mid \frac{f\left(s, x_{s-\sigma_{1 s}}, x_{s-\sigma_{2 s}}, \ldots, x_{s-\sigma_{k s}}\right)}{h\left(x_{i-\tau_{1 i}}, x_{i-\tau_{2 i}}, \ldots, x_{i-\tau_{m i}}\right)}\right. \\
& -\frac{f\left(s, y_{s-\sigma_{1 s}}, y_{s-\sigma_{2 s}}, \ldots, y_{s-\sigma_{k s}}\right)}{h\left(x_{i-\tau_{1 i}}, x_{i-\tau_{2 i}}, \ldots, x_{i-\tau_{m i}}\right)} \mid \\
& +\mid \frac{f\left(s, y_{s-\sigma_{1 s}}, y_{s-\sigma_{2 s}}, \ldots, y_{s-\sigma_{k s}}\right)}{h\left(x_{i-\tau_{1 i}}, x_{i-\tau_{2 i}}, \ldots, x_{i-\tau_{m i}}\right)} \\
& -\frac{f\left(s, y_{s-\sigma_{1 s}}, y_{s-\sigma_{2 s}}, \ldots, y_{s-\sigma_{k s}}\right)}{h\left(y_{i-\tau_{1 i}}, y_{i-\tau_{2 i}}, \ldots, y_{i-\tau_{m i}}\right)}|+| b_{s} \mid \\
& \cdot \mid \frac{1}{h\left(x_{i-\tau_{1 i}}, x_{i-\tau_{2 i}}, \ldots, x_{i-\tau_{m i}}\right)} \\
& \left.-\frac{1}{h\left(y_{i-\tau_{1 i}}, y_{i-\tau_{2 i}}, \ldots, y_{i-\tau_{m i}}\right)} \mid\right] \leq q\|x-y\| \\
& +\sum_{i=n}^{\infty} \frac{1}{\left|a_{i}\right|} \sum_{s=n_{0}}^{i-1}\left[\frac{P_{s}}{m_{h}} \max \left\{\left|x_{s-\sigma_{j s}}-y_{s-\sigma_{j s}}\right|: 1 \leq j \leq k\right\}\right. \\
& +\frac{L_{h}}{m_{h}^{2}}\left(\left|f\left(s, y_{s-\sigma_{1 s}}, y_{s-\sigma_{2 s}}, \ldots, y_{s-\sigma_{k s}}\right)\right|+\left|b_{s}\right|\right) \\
& \left.\cdot \max \left\{\left|x_{i-\tau_{j i}}-y_{i-\tau_{j i}}\right|: 1 \leq j \leq m\right\}\right] \leq q\|x-y\|
\end{aligned}
$$

$$
\begin{aligned}
& +\sum_{i=n}^{\infty} \frac{1}{\left|a_{i}\right|} \sum_{s=n_{0}}^{i-1}\left[\frac{P_{s}}{m_{h}}\|x-y\|+\frac{L_{h}\left(W_{s}+\left|b_{s}\right|\right)}{m_{h}^{2}} \| x\right. \\
& -y \|] \leq\left\{q+\frac{1}{m_{h}^{2}} \sum_{i=D}^{\infty} \frac{1}{\left|a_{i}\right|} \sum_{s=n_{0}}^{i-1}\left[m_{h} P_{s}\right.\right. \\
& \left.\left.+L_{h}\left(W_{s}+\left|b_{s}\right|\right)\right]\right\}\|x-y\|=\theta\|x-y\|,
\end{aligned}
$$

which yield that

$$
\begin{gathered}
T(A(N, M)) \subseteq A(N, M), \\
\|T x-T y\| \leq \theta\|x-y\|,
\end{gathered}
$$

$$
\forall x, y \in A(N, M)
$$

that is, $T$ is a contraction operator in $A(N, M)$ and $T$ has a unique fixed point $x=\left\{x_{n}\right\}_{n \in \mathbb{Z}_{\beta}} \in A(N, M)$, which means that for all $n \geq D$

$$
\begin{aligned}
x_{n}=c & +q_{n} x_{n-\tau_{0}}+\sum_{i=n}^{\infty} \frac{1}{a_{i} h\left(x_{i-\tau_{1 i}}, x_{i-\tau_{2 i}}, \ldots, x_{i-\tau_{m i}}\right)} \\
& \cdot \sum_{s=n_{0}}^{i-1}\left[f\left(s, x_{s-\sigma_{1 s}}, x_{s-\sigma_{2 s}}, \ldots, x_{s-\sigma_{k s}}\right)-b_{s}\right],
\end{aligned}
$$

which yields that for all $n \geq D$

$$
\begin{aligned}
& \Delta\left(x_{n}-q_{n} x_{n-\tau_{0}}\right)=-\frac{1}{a_{n} h\left(x_{n-\tau_{1 n}}, x_{n-\tau_{2 n}}, \ldots, x_{n-\tau_{m n}}\right)} \\
& \quad \cdot \sum_{s=n_{0}}^{n-1}\left[f\left(s, x_{s-\sigma_{1 s}}, x_{s-\sigma_{2 s}}, \ldots, x_{s-\sigma_{k s}}\right)-b_{s}\right], \\
& \Delta\left(a_{n} h\left(x_{n-\tau_{1 n}}, x_{n-\tau_{2 n}}, \ldots, x_{n-\tau_{m n}}\right) \Delta\left(x_{n}-q_{n} x_{n-\tau_{0}}\right)\right) \\
& =-f\left(n, x_{n-\sigma_{1 n}}, x_{n-\sigma_{2 n}}, \ldots, x_{n-\sigma_{k n}}\right)+b_{n},
\end{aligned}
$$

which implies that $x=\left\{x_{n}\right\}_{n \in \mathbb{Z}_{\beta}}$ is a bounded positive solution of (1) in $A(N, M)$. $n \geq D$

By (20), (24), and (26), we infer that for any $\eta \geq 0$ and

$$
\begin{aligned}
& \left|x_{\eta+1, n}-x_{n}\right|=\mid\left(1-\alpha_{\eta}-\beta_{\eta}\right) x_{\eta, n}+\alpha_{\eta}\left\{c+q_{n} x_{\eta, n-\tau_{0}}\right. \\
& +\sum_{i=n}^{\infty} \frac{1}{a_{i} h\left(x_{\eta, i-\tau_{1 i}}, x_{\eta, i-\tau_{2 i}}, \ldots, x_{\eta, i-\tau_{m i}}\right)} \\
& \left.\quad \cdot \sum_{s=n_{0}}^{i-1}\left[f\left(s, x_{\eta, s-\sigma_{1 s}}, x_{\eta, s-\sigma_{2 s}}, \ldots, x_{\eta, s-\sigma_{k s}}\right)-b_{s}\right]\right\}
\end{aligned}
$$




$$
\begin{aligned}
& +\beta_{\eta} \gamma_{\eta, n}-x_{n}\left|\leq\left(1-\alpha_{\eta}-\beta_{\eta}\right)\right| x_{\eta, n}-x_{n} \mid \\
& +\alpha_{\eta}\left|T x_{\eta, n}-T x_{n}\right|+\beta_{\eta}\left|\gamma_{\eta, n}-x_{n}\right| \leq\left(1-\alpha_{\eta}-\beta_{\eta}\right) \\
& \cdot\left\|x_{\eta}-x\right\|+\alpha_{\eta} \theta\left\|x_{\eta}-x\right\|+2 M \beta_{\eta} \leq(1-(1-\theta) \\
& \left.\cdot \alpha_{\eta}\right)\left\|x_{\eta}-x\right\|+2 M \beta_{\eta},
\end{aligned}
$$

which implies that

$$
\left\|x_{\eta+1}-x\right\| \leq\left(1-(1-\theta) \alpha_{\eta}\right)\left\|x_{\eta}-x\right\|+2 M \beta_{\eta}
$$

$\eta \geq 0$.

That is, (21) holds. Consequently, Lemma 1 and (21) (22) imply that $\lim _{\eta \rightarrow \infty} x_{\eta}=x$.
Next, we show that (b) holds. Let $c_{1}, c_{2}$ denote two arbitrary constants in $(N,(1-q) M)$ with $c_{1} \neq c_{2}$. As in the proof of (a), we infer similarly that for each $l \in\{1,2\}$ there exist constants $\theta_{l} \in(0,1), D_{l}>1+n_{0}+\tau_{0}+|\beta|$, and an operator $T_{l}: A(N, M) \rightarrow A(N, M)$ satisfying (23), (24), and (26), where $\theta_{l}, D_{l}$, and $T_{l}$ take the place of $\theta, D$, and $T$, respectively. Moreover, the contraction operator $T_{l}$ has a unique fixed point $x^{l}=\left\{x_{n}^{l}\right\}_{n \in \mathbb{Z}_{\beta}} \in A(N, M)$, which is also a bounded positive solution of (1); that is,

$$
\begin{aligned}
x_{n}^{l}=c_{l} & +q_{n} x_{n-\tau_{0}}^{l}+\sum_{i=n}^{\infty} \frac{1}{a_{i} h\left(x_{i-\tau_{1 i}}^{l}, x_{i-\tau_{2 i}}^{l}, \ldots, x_{i-\tau_{m i}}^{l}\right)} \\
& \cdot \sum_{s=n_{0}}^{i-1}\left[f\left(s, x_{s-\sigma_{1 s}}^{l}, x_{s-\sigma_{2 s}}^{l}, \ldots, x_{s-\sigma_{k s}}^{l}\right)-b_{s}\right],
\end{aligned}
$$

$\forall n \geq D_{l}$,

which together with (15) (18), (23), (24), and (26) gives that for each $n \geq \max \left\{D_{1}, D_{2}\right\}$

$$
\begin{aligned}
& \left|x_{n}^{1}-x_{n}^{2}\right|=\mid c_{1}+q_{n} x_{n-\tau_{0}}^{1}+\sum_{i=n}^{\infty} \frac{1}{a_{i} h\left(x_{i-\tau_{1 i}}^{1}, x_{i-\tau_{2 i}}^{1}, \ldots, x_{i-\tau_{m i}}^{1}\right)} \sum_{s=n_{0}}^{i-1}\left[f\left(s, x_{s-\sigma_{1 s}}^{1}, x_{s-\sigma_{2 s}}^{1}, \ldots, x_{s-\sigma_{k s}}^{1}\right)-b_{s}\right]-c_{2}-q_{n} x_{n-\tau_{0}}^{2} \\
& -\sum_{i=n}^{\infty} \frac{1}{a_{i} h\left(x_{i-\tau_{1 i}}^{2}, x_{i-\tau_{2 i}}^{2}, \ldots, x_{i-\tau_{m i}}^{2}\right)} \sum_{s=n_{0}}^{i-1}\left[f\left(s, x_{s-\sigma_{1 s}}^{2}, x_{s-\sigma_{2 s}}^{2}, \ldots, x_{s-\sigma_{k s}}^{2}\right)-b_{s}\right]|\geq| c_{1}-c_{2}|-| q_{n}|| x_{n-\tau_{0}}^{1}-x_{n-\tau_{0}}^{2} \mid-\sum_{i=n}^{\infty} \frac{1}{\left|a_{i}\right|} \\
& \cdot \sum_{s=n_{0}}^{i-1}\left[\left|\frac{f\left(s, x_{s-\sigma_{1 s}}^{1}, x_{s-\sigma_{2 s}}^{1}, \ldots, x_{s-\sigma_{k s}}^{1}\right)}{h\left(x_{i-\tau_{1 i}}^{1}, x_{i-\tau_{2 i}}^{1}, \ldots, x_{i-\tau_{m i}}^{1}\right)}-\frac{f\left(s, x_{s-\sigma_{1 s}}^{2}, x_{s-\sigma_{2 s}}^{2}, \ldots, x_{s-\sigma_{k s}}^{2}\right)}{h\left(x_{i-\tau_{1 i}}^{2}, x_{i-\tau_{2 i}}^{2}, \ldots, x_{i-\tau_{m i}}^{2}\right)}\right|\right. \\
& \left.+\left|b_{s}\right|\left|\frac{1}{h\left(x_{i-\tau_{1 i}}^{1}, x_{i-\tau_{2 i}}^{1}, \ldots, x_{i-\tau_{m i}}^{1}\right)}-\frac{1}{h\left(x_{i-\tau_{1 i}}^{2}, x_{i-\tau_{2 i}}^{2}, \ldots, x_{i-\tau_{m i}}^{2}\right)}\right|\right] \geq\left|c_{1}-c_{2}\right|-q\left\|x^{1}-x^{2}\right\|-\sum_{i=n}^{\infty} \frac{1}{\left|a_{i}\right|} \\
& \cdot \sum_{s=n_{0}}^{i-1}\left[\left|\frac{f\left(s, x_{s-\sigma_{1 s}}^{1}, x_{s-\sigma_{2 s}}^{1}, \ldots, x_{s-\sigma_{k s}}^{1}\right)-f\left(s, x_{s-\sigma_{1 s}}^{2}, x_{s-\sigma_{2 s}}^{2}, \ldots, x_{s-\sigma_{k s}}^{2}\right)}{h\left(x_{i-\tau_{1 i}}^{1}, x_{i-\tau_{2 i}}^{1}, \ldots, x_{i-\tau_{m i}}^{1}\right)}\right|\right. \\
& +\left|\frac{f\left(s, x_{s-\sigma_{1 s}}^{2}, x_{s-\sigma_{2 s}}^{2}, \ldots, x_{s-\sigma_{k s}}^{2}\right)}{h\left(x_{i-\tau_{1 i}}^{1}, x_{i-\tau_{2 i}}^{1}, \ldots, x_{i-\tau_{m i}}^{1}\right)}-\frac{f\left(s, x_{s-\sigma_{1 s}}^{2}, x_{s-\sigma_{2 s}}^{2}, \ldots, x_{s-\sigma_{k s}}^{2}\right)}{h\left(x_{i-\tau_{1 i}}^{2}, x_{i-\tau_{2 i}}^{2}, \ldots, x_{i-\tau_{m i}}^{2}\right)}\right| \\
& \left.+\frac{\left|b_{s}\right|}{m_{h}^{2}}\left|h\left(x_{i-\tau_{1 i}}^{1}, x_{i-\tau_{2 i}}^{1}, \ldots, x_{i-\tau_{m i}}^{1}\right)-h\left(x_{i-\tau_{1 i}}^{2}, x_{i-\tau_{2 i}}^{2}, \ldots, x_{i-\tau_{m i}}^{2}\right)\right|\right] \geq\left|c_{1}-c_{2}\right|-q\left\|x^{1}-x^{2}\right\|-\sum_{i=n}^{\infty} \frac{1}{\left|a_{i}\right|} \\
& \cdot \sum_{s=n_{0}}^{i-1}\left[\frac{P_{s}}{m_{h}} \max \left\{\left|x_{s-\sigma_{l s}}^{1}-x_{s-\sigma_{l s}}^{2}\right|: 1 \leq l \leq k\right\}+\frac{L_{h}\left(W_{s}+\left|b_{s}\right|\right)}{m_{h}^{2}} \max \left\{\left|x_{i-\tau_{l i}}^{1}-x_{i-\tau_{l i}}^{2}\right|: 1 \leq l \leq m\right\}\right] \geq\left|c_{1}-c_{2}\right|-q \| x^{1} \\
& -x^{2} \|-\frac{\left\|x^{1}-x^{2}\right\|}{m_{h}^{2}} \sum_{i=n}^{\infty} \frac{1}{\left|a_{i}\right|} \sum_{s=n_{0}}^{i-1}\left[m_{h} P_{s}+L_{h}\left(W_{s}+\left|b_{s}\right|\right)\right] \geq\left|c_{1}-c_{2}\right|-\left\{q+\frac{1}{m_{h i=\max \left\{D_{1}, D_{2}\right\}}^{2}} \sum_{\left|a_{i}\right|}^{\infty}\right. \\
& \left.\cdot \sum_{s=n_{0}}^{i-1}\left[m_{h} P_{s}+L_{h}\left(W_{s}+\left|b_{s}\right|\right)\right]\right\}\left\|x^{1}-x^{2}\right\| \geq\left|c_{1}-c_{2}\right|-\max \left\{\theta_{1}, \theta_{2}\right\}\left\|x^{1}-x^{2}\right\|,
\end{aligned}
$$


which yields that

$$
\left\|x^{1}-x^{2}\right\| \geq \frac{\left|c_{1}-c_{2}\right|}{1+\max \left\{\theta_{1}, \theta_{2}\right\}}>0 ;
$$

that is, $x^{1} \neq x^{2}$. This completes the proof.

Theorem 4. Assume that there exist constants $M, N, q$, $L_{h}$, and $m_{h}$ and two nonnegative sequences $\left\{P_{n}\right\}_{n \in \mathbb{N}_{n_{0}}}$ and $\left\{W_{n}\right\}_{n \in \mathbb{N}_{n_{0}}}$ satisfying (15) (19):

$$
\begin{aligned}
0 & <N<(1+q) M ; \\
-1 & <q \leq q_{n} \leq 0, \quad \text { eventually. }
\end{aligned}
$$

Then, one has the following.

(a) For any $c \in(N-q M, M)$, there exist $\theta \in(0,1)$ and $D \geq 1+n_{0}+\tau_{0}+|\beta|$ such that, for each $x_{0}=$ $\left\{x_{0, n}\right\}_{n \in \mathbb{Z}_{\beta}} \in A(N, M)$, the Mann iterative sequence with errors $\left\{x_{\eta}\right\}_{\eta \in \mathbb{N}_{0}}=\left\{x_{\eta, n}\right\}_{(\eta, n) \in \mathbb{N}_{0} \times \mathbb{Z}_{\beta}}$ generated by (20) converges to a bounded positive solution $x \in A(N, M)$ of (1) and has the error estimate (21), where $\left\{\gamma_{\eta}\right\}_{\eta \in \mathbb{N}_{0}}=\left\{\gamma_{\eta, n}\right\}_{(\eta, n) \in \mathbb{N}_{0} \times \mathbb{Z}_{\beta}}$ is an arbitrary sequence in $A(N, M)$ and $\left\{\alpha_{\eta}\right\}_{\eta \in \mathbb{N}_{0}}$ and $\left\{\beta_{\eta}\right\}_{\eta \in \mathbb{N}_{0}}$ are any sequences in $[0,1]$ satisfying $(22)$.

(b) Equation (1) has uncountably many bounded positive solutions in $A(N, M)$.

Proof. First, we show that (a) holds. Let $c \in(N-q M, M)$. It follows from (19) and (35) that there exist $\theta \in(0,1)$ and $D \geq 1+n_{0}+\tau_{0}+|\beta|$ sufficiently large such that

$$
\begin{aligned}
& -1<q \leq q_{n} \leq 0, \quad \forall n \geq D, \\
& \theta=-q+\frac{1}{m_{h}^{2}} \sum_{i=D}^{\infty} \frac{1}{\left|a_{i}\right|} \sum_{s=n_{0}}^{i-1}\left[m_{h} P_{s}+L_{h}\left(W_{s}+\left|b_{s}\right|\right)\right], \\
& \frac{1}{m_{h}} \sum_{i=D}^{\infty} \frac{1}{\left|a_{i}\right|} \sum_{s=n_{0}}^{i-1}\left(W_{s}+\left|b_{s}\right|\right) \\
& \quad<\min \{c+q M-N, M-c\} .
\end{aligned}
$$

Let the operator $T: A(N, M) \rightarrow l_{\beta}^{\infty}$ be defined by (24). By virtue of (15) (18), (24), and (36), we obtain that, for every $x=\left\{x_{n}\right\}_{n \in \mathbb{Z}_{\beta}}, y=\left\{y_{n}\right\}_{n \in \mathbb{Z}_{\beta}} \in A(N, M)$, and $n \geq D$,

$$
\begin{gathered}
T x_{n}=c+q_{n} x_{n-\tau_{0}}+\sum_{i=n}^{\infty} \frac{1}{a_{i} h\left(x_{i-\tau_{1 i}}, x_{i-\tau_{2 i}}, \ldots, x_{i-\tau_{m i}}\right)} \\
\cdot \sum_{s=n_{0}}^{i-1}\left[f\left(s, x_{s-\sigma_{1 s}}, x_{s-\sigma_{2 s}}, \ldots, x_{s-\sigma_{k s}}\right)-b_{s}\right] \geq c
\end{gathered}
$$

$$
\begin{aligned}
+ & q M-\frac{1}{m_{h}} \sum_{i=n}^{\infty} \frac{1}{\left|a_{i}\right|} \\
\cdot & \sum_{s=n_{0}}^{i-1}\left[\left|f\left(s, x_{s-\sigma_{1 s}}, x_{s-\sigma_{2 s}}, \ldots, x_{s-\sigma_{k s}}\right)\right|+\left|b_{s}\right|\right] \geq c \\
+ & q M-\frac{1}{m_{h}} \sum_{i=n}^{\infty} \frac{1}{\left|a_{i}\right|} \sum_{s=n_{0}}^{i-1}\left(W_{s}+\left|b_{s}\right|\right) \geq c+q M \\
& -\min \{c+q M-N, M-c\} \geq N, \\
T x_{n} & =c+q_{n} x_{n-\tau_{0}}+\sum_{i=n}^{\infty} \frac{1}{a_{i} h\left(x_{i-\tau_{1 i}}, x_{i-\tau_{2 i}}, \ldots, x_{i-\tau_{m i}}\right)} \\
& \cdot \sum_{s=n_{0}}^{i-1}\left[f\left(s, x_{s-\sigma_{1 s}}, x_{s-\sigma_{2 s}}, \ldots, x_{s-\sigma_{k s}}\right)-b_{s}\right] \leq c \\
& +\frac{1}{m_{h}} \sum_{i=n}^{\infty} \frac{1}{\left|a_{i}\right|} \\
& \cdot \sum_{s=n_{0}}^{i-1}\left[\left|f\left(s, x_{s-\sigma_{1 s}}, x_{s-\sigma_{2 s}}, \ldots, x_{s-\sigma_{k s}}\right)\right|+\left|b_{s}\right|\right] \leq c \\
& +\frac{1}{m_{h}} \sum_{i=n}^{\infty} \frac{1}{\left|a_{i}\right|} \sum_{s=n_{0}}^{i-1}\left(W_{s}+\left|b_{s}\right|\right) \leq c \\
& +\min \{c+q M-N, M-c\} \leq M .
\end{aligned}
$$

The rest of the proof is similar to that of Theorem 3 and is omitted. This completes the proof.

Theorem 5. Assume that there exist constants $M, N, \underline{q}, \bar{q}$, $L_{h}$, and $m_{h}$ and two nonnegative sequences $\left\{P_{n}\right\}_{n \in \mathbb{N}_{n_{0}}}$ and $\left\{W_{n}\right\}_{n \in \mathbb{N}_{n_{0}}}$ satisfying (15) (19):

$$
\begin{aligned}
& 0<\bar{q} N<(\underline{q}-1) M \\
& 1<\underline{q} \leq q_{n} \leq \bar{q}, \quad \text { eventually. }
\end{aligned}
$$

Then, one has the following.

(a) For any $c \in(\bar{q} N,(\underline{q}-1) M)$, there exist $\theta \in(0,1)$ and $D \geq 1+n_{0}+\tau_{0}+|\beta|$ such that, for each $x_{0}=\left\{x_{0, n}\right\}_{n \in \mathbb{Z}_{\beta}} \epsilon$ $A(N, M)$, the Mann iterative sequence with errors $\left\{x_{\eta}\right\}_{\eta \in \mathbb{N}_{0}}=$ $\left\{x_{\eta, n}\right\}_{(\eta, n) \in \mathbb{N}_{0} \times \mathbb{Z}_{\beta}}$ generated by the scheme

$$
\begin{aligned}
& x_{\eta+1, n} \\
& =\left\{\begin{array}{l}
\left(1-\alpha_{\eta}-\beta_{\eta}\right) x_{\eta, n}+\alpha_{\eta}\left\{\frac{c}{q_{n+\tau_{0}}}+\frac{x_{\eta, n+\tau_{0}}}{q_{n+\tau_{0}}}-\frac{1}{q_{n+\tau_{0}}} \sum_{i=n+\tau_{0}}^{\infty} \frac{1}{a_{i} h\left(x_{\eta, i-\tau_{1 i}}, x_{\eta, i-\tau_{2 i}}, \ldots, x_{\eta, i-\tau_{m i}}\right)} \sum_{s=n_{0}}^{i-1}\left[f\left(s, x_{\eta, s-\sigma_{1 s}}, x_{\eta, s-\sigma_{2 s}}, \ldots, x_{\eta, s-\sigma_{k s}}\right)-b_{s}\right]\right\}+\beta_{\eta} \gamma_{\eta, n}, \quad n \geq D, \eta \geq 0, \\
\left(1-\alpha_{\eta}-\beta_{\eta}\right) x_{\eta, D}+\alpha_{\eta}\left\{\frac{c}{q_{D+\tau_{0}}}+\frac{x_{\eta, D+\tau_{0}}}{q_{D+\tau_{0}}}-\frac{1}{q_{D+\tau_{0}}} \sum_{i=D+\tau_{0}}^{\infty} \frac{1}{a_{i} h\left(x_{\eta, i-\tau_{1 i}}, x_{\eta, i-\tau_{2 i}}, \ldots, x_{\eta, i-\tau_{m i}}\right)} \sum_{s=n_{0}}^{i-1}\left[f\left(s, x_{\eta, s-\sigma_{1 s}}, x_{\eta, s-\sigma_{2 s}}, \ldots, x_{\eta, s-\sigma_{k s}}\right)-b_{s}\right]\right\}+\beta_{\eta} \gamma_{\eta, D}, \quad \beta \leq n<D, \eta \geq 0,
\end{array}\right.
\end{aligned}
$$

converges to a bounded positive solution $x \in A(N, M)$ of (1) and has the error estimate (21), where $\left\{\gamma_{\eta}\right\}_{\eta \in \mathbb{N}_{0}}=\left\{\gamma_{\eta, n}\right\}_{(\eta, n) \in \mathbb{N}_{0} \times \mathbb{Z}_{\beta}}$ is an arbitrary sequence in $A(N, M)$ and $\left\{\alpha_{\eta}\right\}_{\eta \in \mathbb{N}_{0}}$ and $\left\{\beta_{\eta}\right\}_{\eta \in \mathbb{N}_{0}}$ are any sequences in $[0,1]$ satisfying (22). 
(b) Equation (1) has uncountably many bounded positive solutions in $A(N, M)$.

Proof. First, we show that (a) holds. Let $c \in(\bar{q} N,(q-1) M)$. It follows from (19) and (39) that there exist $\theta \in \overline{(0,1)}$ and $D \geq 1+n_{0}+\tau_{0}+|\beta|$ sufficiently large such that

$$
\begin{aligned}
\frac{1}{m_{h}} \sum_{i=D}^{\infty} \frac{1}{\left|a_{i}\right|} \sum_{s=n_{0}}^{i-1}\left(W_{s}+\left|b_{s}\right|\right) \\
\quad<\min \left\{\left(\frac{c}{\bar{q}}-N\right) \underline{q}, \underline{q} M-c-M\right\} .
\end{aligned}
$$

$$
\begin{aligned}
& 1<\underline{q} \leq q_{n} \leq \bar{q}, \quad \forall n \geq D ; \\
& \theta=\frac{1}{\underline{q}}+\frac{1}{\underline{q} m_{h}^{2}} \sum_{i=D}^{\infty} \frac{1}{\left|a_{i}\right|} \sum_{s=n_{0}}^{i-1}\left[m_{h} P_{s}+L_{h}\left(W_{s}+\left|b_{s}\right|\right)\right] ;
\end{aligned}
$$

Define an operator $T: A(N, M) \rightarrow l_{\beta}^{\infty}$ as follows:

$$
T x_{n}= \begin{cases}\frac{c}{q_{n+\tau_{0}}}+\frac{x_{n+\tau_{0}}}{q_{n+\tau_{0}}}-\frac{1}{q_{n+\tau_{0}}} \sum_{i=n+\tau_{0}}^{\infty} \frac{1}{a_{i} h\left(x_{i-\tau_{1 i}}, x_{i-\tau_{2 i}}, \ldots, x_{i-\tau_{m i}}\right)} \sum_{s=n_{0}}^{i-1}\left[f\left(s, x_{s-\sigma_{1 s}}, x_{s-\sigma_{2 s}}, \ldots, x_{s-\sigma_{k s}}\right)-b_{s}\right], & n \geq D, \\ T x_{D}, & \beta \leq n<D,\end{cases}
$$

for any $x=\left\{x_{n}\right\}_{n \in \mathbb{Z}_{\beta}} \in A(N, M)$. In view of (15) (18) and (41) (42), we get that, for every $x=\left\{x_{n}\right\}_{n \in \mathbb{Z}_{\beta}}, y=\left\{y_{n}\right\}_{n \in \mathbb{Z}_{\beta}} \in$ $A(N, M)$, and $n \geq D$,

$$
\begin{aligned}
& T x_{n}=\frac{c}{q_{n+\tau_{0}}}+\frac{x_{n+\tau_{0}}}{q_{n+\tau_{0}}}-\frac{1}{q_{n+\tau_{0}}} \\
& \sum_{i=n+\tau_{0}}^{\infty} \frac{1}{a_{i} h\left(x_{i-\tau_{1 i}}, x_{i-\tau_{2 i}}, \ldots, x_{i-\tau_{m i}}\right)} \\
& \cdot \sum_{s=n_{0}}^{i-1}\left[f\left(s, x_{s-\sigma_{1 s}}, x_{s-\sigma_{2 s}}, \ldots, x_{s-\sigma_{k s}}\right)-b_{s}\right] \geq \frac{c}{\bar{q}} \\
& -\frac{1}{q m_{h}} \sum_{i=n+\tau_{0}}^{\infty} \frac{1}{\left|a_{i}\right|} \\
& \cdot \sum_{s=n_{0}}^{i-1}\left[\left|f\left(s, x_{s-\sigma_{1 s}}, x_{s-\sigma_{2 s}}, \ldots, x_{s-\sigma_{k s}}\right)\right|+\left|b_{s}\right|\right] \geq \frac{c}{\bar{q}} \\
& -\frac{1}{\underline{q} m_{h}} \sum_{i=n+\tau_{0}}^{\infty} \frac{1}{\left|a_{i}\right|} \sum_{s=n_{0}}^{i-1}\left(W_{s}+\left|b_{s}\right|\right) \geq \frac{c}{\bar{q}}-\frac{1}{\underline{q}} \min \left\{\left(\frac{c}{\bar{q}}\right.\right. \\
& -N) \underline{q}, \underline{q} M-c-M\} \geq N, \\
& T x_{n}=\frac{c}{q_{n+\tau_{0}}}+\frac{x_{n+\tau_{0}}}{q_{n+\tau_{0}}}-\frac{1}{q_{n+\tau_{0}}} \\
& \cdot \sum_{i=n+\tau_{0}}^{\infty} \frac{1}{a_{i} h\left(x_{i-\tau_{1 i}}, x_{i-\tau_{2 i}}, \ldots, x_{i-\tau_{m i}}\right)} \\
& \cdot \sum_{s=n_{0}}^{i-1}\left[f\left(s, x_{s-\sigma_{1 s}}, x_{s-\sigma_{2 s}}, \ldots, x_{s-\sigma_{k s}}\right)-b_{s}\right] \leq \frac{c}{\underline{q}} \\
& +\frac{M}{\underline{q}}+\frac{1}{\underline{q} m_{h}} \sum_{i=n+\tau_{0}}^{\infty} \frac{1}{\left|a_{i}\right|}
\end{aligned}
$$

$$
\cdot \sum_{s=n_{0}}^{i-1}\left[\left|f\left(s, x_{s-\sigma_{1 s}}, x_{s-\sigma_{2 s}}, \ldots, x_{s-\sigma_{k s}}\right)\right|+\left|b_{s}\right|\right] \leq \frac{c}{\underline{q}}
$$$$
+\frac{M}{\underline{q}}+\frac{1}{\underline{q} m_{h}} \sum_{i=n+\tau_{0}}^{\infty} \frac{1}{\left|a_{i}\right|} \sum_{s=n_{0}}^{i-1}\left(W_{s}+\left|b_{s}\right|\right) \leq \frac{c}{q}+\frac{M}{\underline{q}}
$$$$
+\frac{1}{q} \min \left\{\left(\frac{c}{\bar{q}}-N\right) \underline{q}, \underline{q} M-c-M\right\} \leq M,
$$$$
\left|T x_{n}-T y_{n}\right|=\mid \frac{x_{n+\tau_{0}}-y_{n+\tau_{0}}}{q_{n+\tau_{0}}}-\frac{1}{q_{n+\tau_{0}}}
$$$$
\cdot \sum_{i=n+\tau_{0}}^{\infty} \frac{1}{a_{i} h\left(x_{i-\tau_{1 i}}, x_{i-\tau_{2 i}}, \ldots, x_{i-\tau_{m i}}\right)}
$$$$
\cdot \sum_{s=n_{0}}^{i-1}\left[f\left(s, x_{s-\sigma_{1 s}}, x_{s-\sigma_{2 s}}, \ldots, x_{s-\sigma_{k s}}\right)-b_{s}\right]+\frac{1}{q_{n+\tau_{0}}}
$$$$
\cdot \sum_{i=n+\tau_{0}}^{\infty} \frac{1}{a_{i} h\left(y_{i-\tau_{1 i}}, y_{i-\tau_{2 i}}, \ldots, y_{i-\tau_{m i}}\right)}
$$$$
\cdot \sum_{s=n_{0}}^{i-1}\left[f\left(s, y_{s-\sigma_{1 s}}, y_{s-\sigma_{2 s}}, \ldots, y_{s-\sigma_{k s}}\right)-b_{s}\right] \mid \leq \frac{1}{\underline{q}} \| x
$$$$
-y \|+\frac{1}{\underline{q}} \sum_{i=n+\tau_{0}}^{\infty} \frac{1}{\left|a_{i}\right|}
$$$$
\cdot \sum_{s=n_{0}}^{i-1}\left[\mid \frac{f\left(s, x_{s-\sigma_{1 s}}, x_{s-\sigma_{2 s}}, \ldots, x_{s-\sigma_{k s}}\right)}{h\left(x_{i-\tau_{1 i}}, x_{i-\tau_{2 i}}, \ldots, x_{i-\tau_{m i}}\right)}\right.
$$$$
-\frac{f\left(s, y_{s-\sigma_{1 s}}, y_{s-\sigma_{2 s}}, \ldots, y_{s-\sigma_{k s}}\right)}{h\left(x_{i-\tau_{1 i}}, x_{i-\tau_{2 i}}, \ldots, x_{i-\tau_{m i}}\right)} \mid
$$$$
+\mid \frac{f\left(s, y_{s-\sigma_{1 s}}, y_{s-\sigma_{2 s}}, \ldots, y_{s-\sigma_{k s}}\right)}{h\left(x_{i-\tau_{1 i}}, x_{i-\tau_{2 i}}, \ldots, x_{i-\tau_{m i}}\right)}
$$ 


$$
\begin{aligned}
& -\frac{f\left(s, y_{s-\sigma_{1 s}}, y_{s-\sigma_{2 s}}, \ldots, y_{s-\sigma_{k s}}\right)}{h\left(y_{i-\tau_{1 i}}, y_{i-\tau_{2 i}}, \ldots, y_{i-\tau_{m i}}\right)}|+| b_{s} \mid \\
& \cdot \mid \frac{1}{h\left(x_{i-\tau_{1 i}}, x_{i-\tau_{2 i}}, \ldots, x_{i-\tau_{m i}}\right)} \\
& \left.-\frac{1}{h\left(y_{i-\tau_{1 i}}, y_{i-\tau_{2 i}}, \ldots, y_{i-\tau_{m i}}\right)} \mid\right] \leq \frac{1}{q}\|x-y\|+\frac{1}{\underline{q}} \\
& \cdot \sum_{i=n+\tau_{0}}^{\infty} \frac{1}{\left|a_{i}\right|} \sum_{s=n_{0}}^{i-1}\left[\frac{P_{s}}{m_{h}}\right. \\
& \cdot \max \left\{\left|x_{s-\sigma_{j s}}-y_{s-\sigma_{j s}}\right|: 1 \leq j \leq k\right\} \\
& +\frac{L_{h}\left(W_{s}+\left|b_{s}\right|\right)}{m_{h}^{2}} \\
& \left.\cdot \max \left\{\left|x_{s-\tau_{j s}}-y_{s-\tau_{j s}}\right|: 1 \leq j \leq m\right\}\right] \leq\left\{\frac{1}{\underline{q}}\right. \\
& \left.+\frac{1}{q m_{h}^{2}} \sum_{i=n}^{\infty} \frac{1}{\left|a_{i}\right|} \sum_{s=n_{0}}^{i-1}\left[m_{h} P_{s}+L_{h}\left(W_{s}+\left|b_{s}\right|\right)\right]\right\}\|x-y\| \\
& \leq \theta\|x-y\|,
\end{aligned}
$$

which yield that (26) holds; that is, $T$ is a contraction operator in $A(N, M)$ and $T$ has a unique fixed point $x=\left\{x_{n}\right\}_{n \in \mathbb{Z}_{\beta}} \in$ $A(N, M)$, which gives that for all $n \geq D$

$$
\begin{aligned}
x_{n}= & \frac{c}{q_{n+\tau_{0}}}+\frac{x_{n+\tau_{0}}}{q_{n+\tau_{0}}}-\frac{1}{q_{n+\tau_{0}}} \\
& \cdot \sum_{i=n+\tau_{0}}^{\infty} \frac{1}{a_{i} h\left(x_{i-\tau_{1 i}}, x_{i-\tau_{2 i}}, \ldots, x_{i-\tau_{m i}}\right)} \\
& \cdot \sum_{s=n_{0}}^{i-1}\left[f\left(s, x_{s-\sigma_{1 s}}, x_{s-\sigma_{2 s}}, \ldots, x_{s-\sigma_{k s}}\right)-b_{s}\right],
\end{aligned}
$$

which implies that for all $n \geq D$

$$
\begin{aligned}
& \Delta\left(x_{n}-q_{n} x_{n-\tau_{0}}\right)=-\frac{1}{a_{n} h\left(x_{n-\tau_{1 n}}, x_{n-\tau_{2 n}}, \ldots, x_{n-\tau_{m n}}\right)} \\
& \quad \cdot \sum_{s=n_{0}}^{n-1}\left[f\left(s, x_{s-\sigma_{1 s}}, x_{s-\sigma_{2 s}}, \ldots, x_{s-\sigma_{k s}}\right)-b_{s}\right], \\
& \Delta\left(a_{n} h\left(x_{n-\tau_{1 n}}, x_{n-\tau_{2 n}}, \ldots, x_{n-\tau_{m n}}\right) \Delta\left(x_{n}-q_{n} x_{n-\tau_{0}}\right)\right) \\
& \quad=-f\left(n, x_{n-\sigma_{1 n}}, x_{n-\sigma_{2 n}}, \ldots, x_{n-\sigma_{k n}}\right)+b_{n},
\end{aligned}
$$

which means that $x=\left\{x_{n}\right\}_{n \in \mathbb{Z}_{\beta}}$ is a bounded positive solution of (1) in $A(N, M)$.

It follows from (26), (40), and (42) that for any $\eta \geq 0$ and $n \geq D$

$$
\begin{aligned}
& \left|x_{\eta+1, n}-x_{n}\right|=\mid\left(1-\alpha_{\eta}-\beta_{\eta}\right) x_{\eta, n}+\alpha_{\eta}\left\{\frac{c}{q_{n+\tau_{0}}}\right. \\
& +\frac{x_{\eta, n+\tau_{0}}}{q_{n+\tau_{0}}}-\frac{1}{q_{n+\tau_{0}}} \\
& \quad \sum_{i=n+\tau_{0}}^{\infty} \frac{1}{a_{i} h\left(x_{\eta, i-\tau_{1 i}}, x_{\eta, i-\tau_{2 i}}, \ldots, x_{\eta, i-\tau_{m i}}\right)} \\
& \left.\quad \sum_{s=n_{0}}^{i-1}\left[f\left(s, x_{\eta, s-\sigma_{1 s}}, x_{\eta, s-\sigma_{2 s}}, \ldots, x_{\eta, s-\sigma_{k s}}\right)-b_{s}\right]\right\} \\
& +\beta_{\eta} \gamma_{\eta, n}-x_{n}\left|\leq\left(1-\alpha_{\eta}-\beta_{\eta}\right)\right| x_{\eta, n}-x_{n} \mid \\
& +\alpha_{\eta}\left|T x_{\eta, n}-T x_{n}\right|+\beta_{\eta}\left|\gamma_{\eta, n}-x_{n}\right| \leq\left(1-\alpha_{\eta}-\beta_{\eta}\right) \\
& .\left\|x_{\eta}-x\right\|+\alpha_{\eta} \theta\left\|x_{\eta}-x\right\|+2 M \beta_{\eta} \leq(1-(1-\theta) \\
& \left.+\alpha_{\eta}\right)\left\|x_{\eta}-x\right\|+2 M \beta_{\eta},
\end{aligned}
$$

which implies that

$$
\begin{aligned}
&\left\|x_{\eta+1}-x\right\| \leq\left(1-(1-\theta) \alpha_{\eta}\right)\left\|x_{\eta}-x\right\|+2 M \beta_{\eta}, \\
& \eta \geq 0 .
\end{aligned}
$$

That is, (21) holds. Consequently, Lemma 1 and (21) (22) imply that $\lim _{\eta \rightarrow \infty} x_{\eta}=x$.

Next, we show that (b) holds. Let $c_{1}, c_{2} \in(\bar{q} N,(\underline{q}-1) M)$ with $c_{1} \neq c_{2}$. As in the proof of (a), we conclude similarly that for each $l \in\{1,2\}$ there exist constants $\theta_{l} \in(0,1), D_{l}>$ $1+n_{0}+\tau_{0}+|\beta|$, and an operator $T_{l}: A(N, \mathrm{M}) \rightarrow A(N, M)$ satisfying (26) and (41) (42), where $\theta, D$, and $T$ are replaced by $\theta_{l}, D_{l}$, and $T_{l}$, respectively, and the contraction operator $T_{l}$ has a unique fixed point $x^{l}=\left\{x_{n}^{l}\right\}_{n \in \mathbb{Z}_{\beta}} \in A(N, M)$, which is also a bounded positive solution of (1); that is,

$$
\begin{aligned}
& x_{n}^{l}= \frac{c_{l}}{q_{n+\tau_{0}}}+\frac{x_{n+\tau_{0}}^{l}}{q_{n+\tau_{0}}}-\frac{1}{q_{n+\tau_{0}}} \\
& \cdot \sum_{i=n+\tau_{0}}^{\infty} \frac{1}{a_{i} h\left(x_{i-\tau_{1 i}}^{l}, x_{i-\tau_{2 i}}^{l}, \ldots, x_{i-\tau_{m i}}^{l}\right)} \\
& \cdot \sum_{s=n_{0}}^{i-1}\left[f\left(s, x_{s-\sigma_{1 s}}^{l}, x_{s-\sigma_{2 s}}^{l}, \ldots, x_{s-\sigma_{k s}}^{l}\right)-b_{s}\right], \\
& \quad \forall n \geq D_{l},
\end{aligned}
$$

which together with (15) (18), (26), and (41) (42) guarantees that for each $n \geq \max \left\{D_{1}, D_{2}\right\}$ 


$$
\begin{aligned}
& \left|x^{1}-x^{2}\right|=\mid \frac{c_{1}}{q_{n+\tau_{0}}}+\frac{x_{n+\tau_{0}}^{1}}{q_{n+\tau_{0}}}-\frac{1}{q_{n+\tau_{0}}} \sum_{i=n+\tau_{0}}^{\infty} \frac{1}{a_{i} h\left(x_{i-\tau_{1 i}}^{1}, x_{i-\tau_{2 i}}^{1}, \ldots, x_{i-\tau_{m i}}^{1}\right)} \sum_{s=n_{0}}^{i-1}\left[f\left(s, x_{s-\sigma_{1 s}}^{1}, x_{s-\sigma_{2 s}}^{1}, \ldots, x_{s-\sigma_{k s}}^{1}\right)-b_{s}\right]-\frac{c_{2}}{q_{n+\tau_{0}}} \\
& -\frac{x_{n+\tau_{0}}^{2}}{q_{n+\tau_{0}}}+\frac{1}{q_{n+\tau_{0}}} \sum_{i=n+\tau_{0}}^{\infty} \frac{1}{a_{i} h\left(x_{i-\tau_{1 i}}^{2}, x_{i-\tau_{2 i}}^{2}, \ldots, x_{i-\tau_{m i}}^{2}\right)} \sum_{s=n_{0}}^{i-1}\left[f\left(s, x_{s-\sigma_{1 s}}^{2}, x_{s-\sigma_{2 s}}^{2}, \ldots, x_{s-\sigma_{k s}}^{2}\right)-b_{s}\right] \mid \geq \frac{\left|c_{1}-c_{2}\right|}{q_{n+\tau_{0}}} \\
& -\frac{\left|x_{n+\tau_{0}}^{1}-x_{n+\tau_{0}}^{2}\right|}{q_{n+\tau_{0}}}-\frac{1}{q_{n+\tau_{0}}} \sum_{i=n+\tau_{0}}^{\infty} \frac{1}{\left|a_{i}\right|} \sum_{s=n_{0}}^{i-1}\left[\left|\frac{f\left(s, x_{s-\sigma_{1 s}}^{1}, x_{s-\sigma_{2 s}}^{1}, \ldots, x_{s-\sigma_{k s}}^{1}\right)}{h\left(x_{i-\tau_{1 i}}^{1}, x_{i-\tau_{2 i}}^{1}, \ldots, x_{i-\tau_{m i}}^{1}\right)}-\frac{f\left(s, x_{s-\sigma_{1 s}}^{2}, x_{s-\sigma_{2 s}}^{2}, \ldots, x_{s-\sigma_{k s}}^{2}\right)}{h\left(x_{i-\tau_{1 i}}^{2}, x_{i-\tau_{2 i}}^{2}, \ldots, x_{i-\tau_{m i}}^{2}\right)}\right|\right. \\
& \left.+\left|b_{s}\right|\left|\frac{1}{h\left(x_{i-\tau_{1 i}}^{1}, x_{i-\tau_{2 i}}^{1}, \ldots, x_{i-\tau_{m i}}^{1}\right)}-\frac{1}{h\left(x_{i-\tau_{1 i}}^{2}, x_{i-\tau_{2 i}}^{2}, \ldots, x_{i-\tau_{m i}}^{2}\right)}\right|\right] \geq \frac{\left|c_{1}-c_{2}\right|}{\bar{q}}-\frac{|| x^{1}-x^{2} \|}{\underline{q}}-\frac{1}{\underline{q}} \sum_{i=n+\tau_{0}}^{\infty} \frac{1}{\left|a_{i}\right|} \\
& \cdot \sum_{s=n_{0}}^{i-1}\left[\left|\frac{f\left(s, x_{s-\sigma_{1 s}}^{1}, x_{s-\sigma_{2 s}}^{1}, \ldots, x_{s-\sigma_{k s}}^{1}\right)-f\left(s, x_{s-\sigma_{1 s}}^{2}, x_{s-\sigma_{2 s}}^{2}, \ldots, x_{s-\sigma_{k s}}^{2}\right)}{h\left(x_{i-\tau_{1 i}}^{1}, x_{i-\tau_{2 i}}^{1}, \ldots, x_{i-\tau_{m i}}^{1}\right)}\right|\right. \\
& +\left|\frac{f\left(s, x_{s-\sigma_{1 s}}^{2}, x_{s-\sigma_{2 s}}^{2}, \ldots, x_{s-\sigma_{k s}}^{2}\right)}{h\left(x_{i-\tau_{1 i}}^{1}, x_{i-\tau_{2 i}}^{1}, \ldots, x_{i-\tau_{m i}}^{1}\right)}-\frac{f\left(s, x_{s-\sigma_{1 s}}^{2}, x_{s-\sigma_{2 s}}^{2}, \ldots, x_{s-\sigma_{k s}}^{2}\right)}{h\left(x_{i-\tau_{1 i}}^{2}, x_{i-\tau_{2 i}}^{2}, \ldots, x_{i-\tau_{m i}}^{2}\right)}\right| \\
& \left.+\frac{\left|b_{s}\right|}{m_{h}^{2}}\left|h\left(x_{i-\tau_{1 i}}^{1}, x_{i-\tau_{2 i}}^{1}, \ldots, x_{i-\tau_{m i}}^{1}\right)-h\left(x_{i-\tau_{1 i}}^{2}, x_{i-\tau_{2 i}}^{2}, \ldots, x_{i-\tau_{m i}}^{2}\right)\right|\right] \geq \frac{\left|c_{1}-c_{2}\right|}{\bar{q}}-\frac{\left\|x^{1}-x^{2}\right\|}{\underline{q}}-\frac{1}{q} \sum_{i=n}^{\infty} \frac{1}{\left|a_{i}\right|} \\
& \cdot \sum_{s=n_{0}}^{i-1}\left[\frac{P_{s}}{m_{h}} \max \left\{\left|x_{s-\sigma_{l s}}^{1}-x_{s-\sigma_{l s}}^{2}\right|: 1 \leq l \leq k\right\}+\frac{L_{h}\left(W_{s}+\left|b_{s}\right|\right)}{m_{h}^{2}} \max \left\{\left|x_{i-\tau_{l i}}^{1}-x_{i-\tau_{l i}}^{2}\right|: 1 \leq l \leq m\right\}\right] \geq \frac{\left|c_{1}-c_{c}\right|}{\bar{q}}-\left\{\frac{1}{\underline{q}}\right. \\
& \left.+\frac{1}{m_{h}^{2} \underline{\underline{q}}} \sum_{i=\max \left\{D_{1}, D_{2}\right\}}^{\infty} \frac{1}{\left|a_{i}\right|} \sum_{s=n_{0}}^{i-1}\left[m_{h} P_{s}+L_{h}\left(W_{s}+\left|b_{s}\right|\right)\right]\right\}\left\|x^{1}-x^{2}\right\| \geq \frac{\left|c_{1}-c_{2}\right|}{\bar{q}}-\max \left\{\theta_{1}, \theta_{2}\right\}\left\|x^{1}-x^{2}\right\|,
\end{aligned}
$$

which yields that

$$
\left\|x^{1}-x^{2}\right\| \geq \frac{\left|c_{1}-c_{2}\right|}{\bar{q}\left(1+\max \left\{\theta_{1}, \theta_{2}\right\}\right)}>0 ;
$$

that is, $x^{1} \neq x^{2}$. This completes the proof.

Theorem 6. Assume that there exist constants $M, N, q, \bar{q}$, $L_{h}$, and $m_{h}$ and two nonnegative sequences $\left\{P_{n}\right\}_{n \in \mathbb{N}_{n_{0}}}$ and $\left\{W_{n}\right\}_{n \in \mathbb{N}_{n_{0}}}$ satisfying (15) (19):

$$
\begin{aligned}
0 & <\underline{q} \bar{q} N<\left(\underline{q}+\bar{q}^{2}\right) M ; \\
-\bar{q}^{2} & <\underline{q} \leq q_{n} \leq \bar{q}<-1, \quad \text { eventually. }
\end{aligned}
$$

Then, one has the following.

(a) For any $c \in(\bar{q} M, q N-q M / \bar{q})$, there exist $\theta \in$ $(0,1)$ and $D \geq 1+n_{0}+\tau_{0}+|\beta|$ such that, for each $x_{0}=$ $\left\{x_{0, n}\right\}_{n \in \mathbb{Z}_{\beta}} \in A(N, M)$, the Mann iterative sequence with errors $\left\{x_{\eta}\right\}_{\eta \in \mathbb{N}_{0}}=\left\{x_{\eta, n}\right\}_{(\eta, n) \in \mathbb{N}_{0} \times \mathbb{Z}_{\beta}}$ generated by (40) converges to a bounded positive solution $x \in A(N, M)$ of (1) and has the error estimate (21), where $\left\{\gamma_{\eta}\right\}_{\eta \in \mathbb{N}_{0}}=\left\{\gamma_{\eta, n}\right\}_{(\eta, n) \in \mathbb{N}_{0} \times \mathbb{Z}_{\beta}}$ is an arbitrary sequence in $A(N, M)$ and $\left\{\alpha_{\eta}\right\}_{\eta \in \mathbb{N}_{0}}$ and $\left\{\beta_{\eta}\right\}_{\eta \in \mathbb{N}_{0}}$ are any sequences in $[0,1]$ satisfying (22).

(b) Equation (1) has uncountably many bounded positive solutions in $A(N, M)$.

Proof. First, we show that (a) holds. Let $c \in(\bar{q} M, q N-q M / \bar{q})$. It follows from (19) and (52) that there exist $\theta \bar{\epsilon}(0, \overline{1})$ and $D \geq 1+n_{0}+\tau_{0}+|\beta|$ satisfying

$$
\begin{array}{r}
\theta=-\frac{1}{\bar{q}}-\frac{1}{\bar{q} m_{h}^{2}} \sum_{i=D}^{\infty} \frac{1}{\left|a_{i}\right|} \sum_{s=n_{0}}^{i-1}\left[m_{h} P_{s}+L_{h}\left(W_{s}+\left|b_{s}\right|\right)\right], \\
-\bar{q}^{2}<\underline{q} \leq q_{n} \leq \bar{q}<-1, \quad \forall n \geq D,
\end{array}
$$

$$
\begin{aligned}
& \frac{1}{m_{h}} \sum_{i=D}^{\infty} \frac{1}{\left|a_{i}\right|} \sum_{s=n_{0}}^{i-1}\left(W_{s}+\left|b_{s}\right|\right) \\
& \quad<\min \left\{\bar{q} N-\frac{\bar{q} c}{\underline{q}}-M, c-\bar{q} M\right\} .
\end{aligned}
$$


Define an operator $T: A(N, M) \rightarrow l_{\beta}^{\infty}$ by (42). In light of (15) (19), (42), and (53), we get that, for every $x=\left\{x_{n}\right\}_{n \in \mathbb{Z}_{\beta}} \in$ $A(N, M), y=\left\{y_{n}\right\}_{n \in \mathbb{Z}_{\beta}} \in A(N, M)$, and $n \geq D+1$,

$$
\begin{aligned}
& T x_{n}=\frac{c}{q_{n+\tau_{0}}}+\frac{x_{n+\tau_{0}}}{q_{n+\tau_{0}}}-\frac{1}{q_{n+\tau_{0}}} \\
& \cdot \sum_{i=n+\tau_{0}}^{\infty} \frac{1}{a_{i} h\left(x_{i-\tau_{1 i}}, x_{i-\tau_{2 i}}, \ldots, x_{i-\tau_{m i}}\right)} \\
& \cdot \sum_{s=n_{0}}^{i-1}\left[f\left(s, x_{s-\sigma_{1 s}}, x_{s-\sigma_{2 s}}, \ldots, x_{s-\sigma_{k s}}\right)-b_{s}\right] \geq \frac{c}{\underline{q}} \\
& +\frac{M}{\bar{q}}+\frac{1}{\bar{q}} \sum_{i=n+\tau_{0}}^{\infty} \frac{1}{\left|a_{i}\right| m_{h}} \\
& \quad \cdot \sum_{s=n_{0}}^{i-1}\left[\left|f\left(s, x_{s-\sigma_{1 s}}, x_{s-\sigma_{2 s}}, \ldots, x_{s-\sigma_{k s}}\right)\right|+\left|b_{s}\right|\right] \geq \frac{c}{q} \\
& \quad+\frac{M}{\bar{q}}+\frac{1}{\bar{q} m_{h}} \sum_{i=n+\tau_{0}}^{\infty} \frac{1}{\left|a_{i}\right|} \sum_{s=n_{0}}^{i-1}\left(W_{s}+\left|b_{s}\right|\right) \geq \frac{c}{q}+\frac{M}{\bar{q}} \\
& \quad+\frac{1}{\bar{q}} \min \left\{\bar{q} N-\frac{\bar{q} c}{\underline{q}}-M, c-\bar{q} M\right\} \geq N, \\
& T x_{n}=\frac{c}{q_{n+\tau_{0}}}+\frac{x_{n+\tau_{0}}}{q_{n+\tau_{0}}}-\frac{1}{q_{n+\tau_{0}}} \\
& \quad \cdot \sum_{i=n+\tau_{0}}^{\infty} \frac{1}{a_{i} h\left(x_{i-\tau_{1 i}}, x_{i-\tau_{2 i}}, \ldots, x_{i-\tau_{m i}}\right)}
\end{aligned}
$$

$$
\begin{aligned}
& \cdot \sum_{s=D}^{i-1}\left[f\left(s, x_{s-\sigma_{1 s}}, x_{s-\sigma_{2 s}}, \ldots, x_{s-\sigma_{k s}}\right)-b_{s}\right] \leq \frac{c}{\bar{q}}-\frac{1}{\bar{q}} \\
& \cdot \sum_{i=n+\tau_{0}}^{\infty} \frac{1}{\left|a_{i}\right| m_{h}} \\
& \cdot \sum_{s=n_{0}}^{i-1}\left[\left|f\left(s, x_{s-\sigma_{1 s}}, x_{s-\sigma_{2 s}}, \ldots, x_{s-\sigma_{k s}}\right)\right|+\left|b_{s}\right|\right] \leq \frac{c}{\bar{q}} \\
& -\frac{1}{\bar{q} m_{h}} \sum_{i=n+\tau_{0}}^{\infty} \frac{1}{\left|a_{i}\right|} \sum_{s=n_{0}}^{i-1}\left(W_{s}+\left|b_{s}\right|\right) \leq \frac{c}{\bar{q}}-\frac{1}{\bar{q}} \\
& \cdot \min \left\{\bar{q} N-\frac{\bar{q} c}{q}-M, c-\bar{q} M\right\} \leq M .
\end{aligned}
$$

The rest of the proof is similar to that of Theorem 5 and is omitted. This completes the proof.

Theorem 7. Assume that there exist constants $M, N, L_{h}$, and $m_{h}$ with $0<N<M$ and two nonnegative sequences $\left\{P_{n}\right\}_{n \in \mathbb{N}_{n_{0}}}$ and $\left\{W_{n}\right\}_{n \in \mathbb{N}_{n_{0}}}$ satisfying (15) (19) and

$$
q_{n}=-1, \text { eventually. }
$$

Then, one has the following.

(a) For any $c \in(N, M)$, there exist $\theta \in(0,1)$ and $D \geq 1+n_{0}+\tau_{0}+|\beta|$ such that, for each $x_{0}=\left\{x_{0, n}\right\}_{n \in \mathbb{Z}_{\beta}} \in$ $A(N, M)$, the Mann iterative sequence with errors $\left\{x_{\eta}\right\}_{\eta \in \mathbb{N}_{0}}=$ $\left\{x_{\eta, n}\right\}_{(\eta, n) \in \mathbb{N}_{0} \times \mathbb{Z}_{\beta}}$ generated by the scheme

$$
=\left\{\begin{array}{l}
\left(1-\alpha_{\eta}-\beta_{\eta}\right) x_{\eta, n}+\alpha_{\eta}\left\{c+\sum_{j=1}^{\infty} \sum_{i=n+(2 j-1) \tau_{0}}^{n+2 j \tau_{0}-1} \frac{1}{a_{i} h\left(x_{\eta, i-\tau_{1 i}}, x_{\eta, i-\tau_{2 i}}, \ldots, x_{\eta, i-\tau_{m i}}\right)} \sum_{s=n_{0}}^{i-1}\left[f\left(s, x_{\eta, s-\sigma_{1 s}}, x_{\eta, s-\sigma_{2 s}}, \ldots, x_{\eta, s-\sigma_{k s}}\right)-b_{s}\right]\right\}+\beta_{\eta} \gamma_{\eta, n}, \quad n \geq D, \eta \geq 0, \\
\left(1-\alpha_{\eta}-\beta_{\eta}\right) x_{\eta, D}+\alpha_{\eta}\left\{c+\sum_{j=1}^{\infty} \sum_{i=D+(2 j-1) \tau_{0}}^{D+2 j \tau_{0}-1} \frac{1}{a_{i} h\left(x_{\eta, i-\tau_{1 i}}, x_{\eta, i-\tau_{2 i}}, \ldots, x_{\eta, i-\tau_{m i}}\right)} \sum_{s=n_{0}}^{i-1}\left[f\left(s, x_{\eta, s-\sigma_{1 s}}, x_{\eta, s-\sigma_{2 s}}, \ldots, x_{\eta, s-\sigma_{k s}}\right)-b_{s}\right]\right\}+\beta_{\eta} \gamma_{\eta, D}, \quad \beta \leq n<D, \eta \geq 0,
\end{array}\right.
$$

converges to a bounded positive solution $x \in A(N, M)$ of (1) and has the error estimate (21), where $\left\{\gamma_{\eta}\right\}_{\eta \in \mathbb{N}_{0}}=\left\{\gamma_{\eta, n}\right\}_{(\eta, n) \in \mathbb{N}_{0} \times \mathbb{Z}_{\beta}}$ is an arbitrary sequence in $A(N, M)$ and $\left\{\alpha_{\eta}\right\}_{\eta \in \mathbb{N}_{0}}$ and $\left\{\beta_{\eta}\right\}_{\eta \in \mathbb{N}_{0}}$ are any sequences in $[0,1]$ satisfying (22).

(b) Equation (1) has uncountably many bounded positive solutions in $A(N, M)$.

$$
\begin{aligned}
& \theta=\frac{1}{m_{h}^{2}} \sum_{i=D}^{\infty} \frac{1}{\left|a_{i}\right|} \sum_{s=n_{0}}^{i-1}\left[m_{h} P_{s}+L_{h}\left(W_{s}+\left|b_{s}\right|\right)\right] \\
& \frac{1}{m_{h}} \sum_{i=D}^{\infty} \frac{1}{\left|a_{i}\right|} \sum_{s=n_{0}}^{i-1}\left(W_{s}+\left|b_{s}\right|\right)<\min \{c-N, M-c\} .
\end{aligned}
$$

Define an operator $T: A(N, M) \rightarrow l_{\beta}^{\infty}$ as follows:
Proof. First, we show that (a) holds. Let $c \in(N, M)$. It follows from (19) that there exist $\theta \in(0,1)$ and $D \geq 1+n_{0}+$ $\tau_{0}+|\beta|$ sufficiently large such that

$$
T x_{n}= \begin{cases}c+\sum_{j=1}^{\infty} \sum_{i=n+(2 j-1) \tau_{0}}^{n+2 j \tau_{0}-1} \frac{1}{a_{i} h\left(x_{i-\tau_{1 i}}, x_{i-\tau_{2 i}}, \ldots, x_{i-\tau_{m i}}\right)} \sum_{s=n_{0}}^{i-1}\left[f\left(s, x_{s-\sigma_{1 s}}, x_{s-\sigma_{2 s}}, \ldots, x_{s-\sigma_{k s}}\right)-b_{s}\right], & n \geq D, \\ T x_{D}, & \beta \leq n<D,\end{cases}
$$


for each $x=\left\{x_{n}\right\}_{n \in \mathbb{Z}_{\beta}} \in A(N, M)$. In view of (15) (18) and (57) (58), we conclude that, for every $x=\left\{x_{n}\right\}_{n \in \mathbb{Z}_{\beta}} \in$ $A(N, M), y=\left\{y_{n}\right\}_{n \in \mathbb{Z}_{\beta}} \in A(N, M)$, and $n \geq D$,

$$
\begin{aligned}
& T x_{n}=c+\sum_{j=1}^{\infty} \sum_{i=n+(2 j-1) \tau_{0}}^{n+2 j \tau_{0}-1} \frac{1}{a_{i} h\left(x_{i-\tau_{1 i}}, x_{i-\tau_{2 i}}, \ldots, x_{i-\tau_{m i}}\right)} \\
& \cdot \sum_{s=n_{0}}^{i-1}\left[f\left(s, x_{s-\sigma_{1 s}}, x_{s-\sigma_{2 s}}, \ldots, x_{s-\sigma_{k s}}\right)-b_{s}\right] \geq c \\
& -\sum_{i=n+\tau_{0}}^{\infty} \frac{1}{\left|a_{i}\right| m_{h}} \sum_{s=n_{0}}^{i-1}\left[\left|f\left(s, x_{s-\sigma_{1 s}}, x_{s-\sigma_{2 s}}, \ldots, x_{s-\sigma_{k s}}\right)\right|\right. \\
& \left.+\left|b_{s}\right|\right] \geq c-\frac{1}{m_{h}} \sum_{i=n+\tau_{0}}^{\infty} \frac{1}{\left|a_{i}\right|} \sum_{s=n_{0}}^{i-1}\left(W_{s}+\left|b_{s}\right|\right) \geq c \\
& -\min \{c-N, M-c\} \geq N, \\
& T x_{n}=c+\sum_{j=1}^{\infty} \sum_{i=n+(2 j-1) \tau_{0}}^{n+2 j \tau_{0}-1} \frac{1}{a_{i} h\left(x_{i-\tau_{1 i}}, x_{i-\tau_{2 i}}, \ldots, x_{i-\tau_{m i}}\right)} \\
& \cdot \sum_{s=n_{0}}^{i-1}\left[f\left(s, x_{s-\sigma_{1 s}}, x_{s-\sigma_{2 s}}, \ldots, x_{s-\sigma_{k s}}\right)-b_{s}\right] \leq c \\
& +\sum_{i=n+\tau_{0}}^{\infty} \frac{1}{\left|a_{i}\right| m_{h}} \sum_{s=n_{0}}^{i-1}\left[\left|f\left(s, x_{s-\sigma_{1 s}}, x_{s-\sigma_{2 s}}, \ldots, x_{s-\sigma_{k s}}\right)\right|\right. \\
& \left.+\left|b_{s}\right|\right] \leq c+\frac{1}{m_{h}} \sum_{i=n+\tau_{0}}^{\infty} \frac{1}{\left|a_{i}\right|} \sum_{s=n_{0}}^{i-1}\left(W_{s}+\left|b_{s}\right|\right) \leq c \\
& +\min \{c-N, M-c\} \leq M, \\
& \left|T x_{n}-T y_{n}\right| \\
& =\mid \sum_{j=1}^{\infty} \sum_{i=n+(2 j-1) \tau_{0}}^{n+2 j \tau_{0}-1} \frac{1}{a_{i} h\left(x_{i-\tau_{1 i}}, x_{i-\tau_{2 i}}, \ldots, x_{i-\tau_{m i}}\right)} \\
& \cdot \sum_{s=n_{0}}^{i-1}\left[f\left(s, x_{s-\sigma_{1 s}}, x_{s-\sigma_{2 s}}, \ldots, x_{s-\sigma_{k s}}\right)-b_{s}\right] \\
& -\sum_{j=1}^{\infty} \sum_{i=n+(2 j-1) \tau_{0}}^{n+2 j \tau_{0}-1} \frac{1}{a_{i} h\left(y_{i-\tau_{1 i}}, y_{i-\tau_{2 i}}, \ldots, y_{i-\tau_{m i}}\right)} \\
& \cdot \sum_{s=n_{0}}^{i-1}\left[f\left(s, y_{s-\sigma_{1 s}}, y_{s-\sigma_{2 s}}, \ldots, y_{s-\sigma_{k s}}\right)-b_{s}\right] \\
& \leq \sum_{j=1}^{\infty} \sum_{i=n+(2 j-1) \tau_{0}}^{n+2 j \tau_{0}-1} \frac{1}{\left|a_{i}\right|} \\
& \cdot \sum_{s=n_{0}}^{i-1}\left[\mid \frac{f\left(s, x_{s-\sigma_{1 s}}, x_{s-\sigma_{2 s}}, \ldots, x_{s-\sigma_{k s}}\right)}{h\left(x_{i-\tau_{1 i}}, x_{i-\tau_{2 i}}, \ldots, x_{i-\tau_{m i}}\right)}\right.
\end{aligned}
$$

$$
\begin{aligned}
& -\frac{f\left(s, y_{s-\sigma_{1 s}}, y_{s-\sigma_{2 s}}, \ldots, y_{s-\sigma_{k s}}\right)}{h\left(x_{i-\tau_{1 i}}, x_{i-\tau_{2 i}}, \ldots, x_{i-\tau_{m i}}\right)} \mid \\
& +\mid \frac{f\left(s, y_{s-\sigma_{1 s}}, y_{s-\sigma_{2 s}}, \ldots, y_{s-\sigma_{k s}}\right)}{h\left(x_{i-\tau_{1 i}}, x_{i-\tau_{2 i}}, \ldots, x_{i-\tau_{m i}}\right)} \\
& -\frac{f\left(s, y_{s-\sigma_{1 s}}, y_{s-\sigma_{2 s}}, \ldots, y_{s-\sigma_{k s}}\right)}{h\left(y_{i-\tau_{1 i}}, y_{i-\tau_{2 i}}, \ldots, y_{i-\tau_{m i}}\right)}|+| b_{s} \mid \\
& \cdot \mid \frac{1}{h\left(x_{i-\tau_{1 i}}, x_{i-\tau_{2 i}}, \ldots, x_{i-\tau_{m i}}\right)} \\
& \left.-\frac{1}{h\left(y_{i-\tau_{1 i}}, y_{i-\tau_{2 i}}, \ldots, y_{i-\tau_{m i}}\right)} \mid\right] \leq \sum_{j=1}^{\infty} \sum_{i=n+(2 j-1) \tau_{0}}^{n+2 j \tau_{0}-1} \frac{1}{\left|a_{i}\right|} \\
& \cdot \sum_{s=n_{0}}^{i-1}\left[\frac{P_{s}}{m_{h}} \max \left\{\left|x_{s-\sigma_{j s}}-y_{s-\sigma_{j s}}\right|: 1 \leq j \leq k\right\}\right. \\
& +\frac{1}{m_{h}^{2}}\left(\left|f\left(s, y_{s-\sigma_{1 s}}, y_{s-\sigma_{2 s}}, \ldots, y_{s-\sigma_{k s}}\right)\right|+\left|b_{s}\right|\right) \\
& \cdot \mid h\left(x_{i-\tau_{1 i}}, x_{i-\tau_{2 i}}, \ldots, x_{i-\tau_{m i}}\right) \\
& \left.-h\left(y_{i-\tau_{1 i}}, y_{i-\tau_{2 i}}, \ldots, y_{i-\tau_{m i}}\right) \mid\right] \leq \sum_{j=1}^{\infty} \sum_{i=n+(2 j-1) \tau_{0}}^{n+2 j \tau_{0}-1} \frac{1}{\left|a_{i}\right|} \\
& \cdot \sum_{s=n_{0}}^{i-1}\left[\frac{P_{s}}{m_{h}}\|x-y\|+\frac{L_{h}\left(W_{s}+\left|b_{s}\right|\right)}{m_{h}^{2}}\right. \\
& \left.\cdot \max \left\{\left|x_{i-\tau_{j i}}-y_{i-\tau_{j i}}\right|: 1 \leq j \leq m\right\}\right] \leq \frac{1}{m_{h}^{2}} \\
& \cdot \sum_{i=n+\tau_{0}}^{\infty} \frac{1}{\left|a_{i}\right|} \sum_{s=n_{0}}^{i-1}\left[m_{h} P_{s}+L_{h}\left(W_{s}+\left|b_{s}\right|\right)\right]\|x-y\| \\
& \leq \theta\|x-y\|,
\end{aligned}
$$

which yield (26); that is, $T$ is a contraction operator in $A(N, M)$ and $T$ has a unique fixed point $x=\left\{x_{n}\right\}_{n \in \mathbb{Z}_{\beta}} \in$ $A(N, M)$, which means that for all $n \geq D$

$$
\begin{aligned}
x_{n}=c & +\sum_{j=1}^{\infty} \sum_{i=n+(2 j-1) \tau_{0}}^{n+2 j \tau_{0}-1} \frac{1}{a_{i} h\left(x_{i-\tau_{1 i}}, x_{i-\tau_{2 i}}, \ldots, x_{i-\tau_{m i}}\right)} \\
& \cdot \sum_{s=n_{0}}^{i-1}\left[f\left(s, x_{s-\sigma_{1 s}}, x_{s-\sigma_{2 s}}, \ldots, x_{s-\sigma_{k s}}\right)-b_{s}\right],
\end{aligned}
$$

which gives that for all $n \geq D$

$$
\begin{aligned}
& x_{n}+x_{n-\tau_{0}}=2 c \\
& +\sum_{j=1}^{\infty} \sum_{i=n+2(j-1) \tau_{0}}^{n+2 j \tau_{0}-1} \frac{1}{a_{i} h\left(x_{i-\tau_{1 i}}, x_{i-\tau_{2 i}}, \ldots, x_{i-\tau_{m i}}\right)}
\end{aligned}
$$




$$
\begin{aligned}
& \sum_{s=n_{0}}^{i-1}\left[f\left(s, x_{s-\sigma_{1 s}}, x_{s-\sigma_{2 s}}, \ldots, x_{s-\sigma_{k s}}\right)-b_{s}\right]=2 c \\
&+\sum_{i=n}^{\infty} \frac{1}{a_{i} h\left(x_{i-\tau_{1 i}}, x_{i-\tau_{2 i}}, \ldots, x_{i-\tau_{m i}}\right)} \\
& \cdot \sum_{s=n_{0}}^{i-1}\left[f\left(s, x_{s-\sigma_{1 s}}, x_{s-\sigma_{2 s}}, \ldots, x_{s-\sigma_{k s}}\right)-b_{s}\right], \\
& \Delta\left(x_{n}+x_{n-\tau_{0}}\right)=-\frac{1}{a_{n} h\left(x_{n-\tau_{1 n}}, x_{n-\tau_{2 n}}, \ldots, x_{n-\tau_{m n}}\right)} \\
& \quad \cdot \sum_{s=n_{0}}^{n-1}\left[f\left(s, x_{s-\sigma_{1 s}}, x_{s-\sigma_{2 s}}, \ldots, x_{s-\sigma_{k s}}\right)-b_{s}\right],
\end{aligned}
$$

which implies that for all $n \geq D$

$$
\begin{gathered}
\Delta\left(a_{n} h\left(x_{n-\tau_{1 n}}, x_{n-\tau_{2 n}}, \ldots, x_{n-\tau_{m n}}\right) \Delta\left(x_{n}+x_{n-\tau_{0}}\right)\right) \\
=-f\left(n, x_{n-\sigma_{1 n}}, x_{n-\sigma_{2 n}}, \ldots, x_{n-\sigma_{k n}}\right)+b_{n}
\end{gathered}
$$

which means that $x=\left\{x_{n}\right\}_{n \in \mathbb{Z}_{\beta}}$ is a bounded positive solution of (1) in $A(N, M)$.

Using (20), (26), and (58), we deduce that for any $\eta \geq 0$ and $n \geq D$

$$
\begin{aligned}
& \left|x_{\eta+1, n}-x_{n}\right|=\mid\left(1-\alpha_{\eta}-\beta_{\eta}\right) x_{\eta, n}+\alpha_{\eta}\{c \\
& +\sum_{j=1}^{\infty} \sum_{i=n+(2 j-1) \tau_{0}}^{n+2 j \tau_{0}-1} \frac{1}{a_{i} h\left(x_{\eta, i-\tau_{1 i}}, x_{\eta, i-\tau_{2 i}}, \ldots, x_{\eta, i-\tau_{m i}}\right)} \\
& \left.\quad \cdot \sum_{s=n_{0}}^{i-1}\left[f\left(s, x_{\eta, s-\sigma_{1 s}}, x_{\eta, s-\sigma_{2 s}}, \ldots, x_{\eta, s-\sigma_{k s}}\right)-b_{s}\right]\right\} \\
& +\beta_{\eta} \gamma_{\eta, n}-x_{n}\left|\leq\left(1-\alpha_{\eta}-\beta_{\eta}\right)\right| x_{\eta, n}-x_{n} \mid \\
& +\alpha_{\eta}\left|T x_{\eta, n}-T x_{n}\right|+\beta_{\eta}\left|\gamma_{\eta, n}-x_{n}\right| \leq\left(1-\alpha_{\eta}-\beta_{\eta}\right) \\
& \quad \cdot\left\|x_{\eta}-x\right\|+\alpha_{\eta} \theta\left\|x_{\eta}-x\right\|+2 M \beta_{\eta} \leq(1-(1-\theta) \\
& \left.\quad \cdot \alpha_{\eta}\right)\left\|x_{\eta}-x\right\|+2 M \beta_{\eta},
\end{aligned}
$$

which implies that

$$
\left\|x_{\eta+1}-x\right\| \leq\left(1-(1-\theta) \alpha_{\eta}\right)\left\|x_{\eta}-x\right\|+2 M \beta_{\eta}
$$$$
\eta \geq 0 \text {. }
$$

That is, (21) holds. Consequently, Lemma 1 and (21) (22) ensure that $\lim _{\eta \rightarrow \infty} x_{\eta}=x$.

Next, we show that (b) holds. Let $c_{1}, c_{2} \in(N, M)$ with $c_{1} \neq c_{2}$. As in the proof of (a), we deduce similarly that for each $l \in\{1,2\}$ there exist constants $\theta_{l} \in(0,1), D_{l}>1+n_{0}+\tau_{0}+|\beta|$, and an operator $T_{l}: A(N, M) \rightarrow A(N, M)$ satisfying (26) and (57) (58), where $\theta, D$, and $T$ are replaced by $\theta_{l}, D_{l}$, and $T_{l}$, respectively, and the contraction operator $T_{l}$ has a unique fixed point $x^{l}=\left\{x_{n}^{l}\right\}_{n \in \mathbb{Z}_{\beta}} \in A(N, M)$, which is also a bounded positive solution of (1); that is,

$$
\begin{array}{r}
x_{n}^{l}=c_{l}+\sum_{j=1}^{\infty} \sum_{i=n+(2 j-1) \tau_{0}}^{n+2 j \tau_{0}-1} \frac{1}{a_{i} h\left(x_{i-\tau_{1 i}}^{l}, x_{i-\tau_{2 i}}^{l}, \ldots, x_{i-\tau_{m i}}^{l}\right)} \\
\cdot \sum_{s=n_{0}}^{i-1}\left[f\left(s, x_{s-\sigma_{1 s}}^{l}, x_{s-\sigma_{2 s}}^{l}, \ldots, x_{s-\sigma_{k s}}^{l}\right)-b_{s}\right], \\
\forall n \geq D_{l},
\end{array}
$$

which together with (15) (18), (26), and (57) (58) means that for each $n \geq \max \left\{D_{1}, D_{2}\right\}$

$$
\begin{aligned}
& \left|x_{n}^{1}-x_{n}^{2}\right|=\mid c_{1} \\
& +\sum_{j=1}^{\infty} \sum_{i=n+(2 j-1) \tau_{0}}^{n+2 j \tau_{0}-1} \frac{1}{a_{i} h\left(x_{i-\tau_{1 i}}^{1}, x_{i-\tau_{2 i}}^{1}, \ldots, x_{i-\tau_{m i}}^{1}\right)} \\
& \cdot \sum_{s=n_{0}}^{i-1}\left[f\left(s, x_{s-\sigma_{1 s}}^{1}, x_{s-\sigma_{2 s}}^{1}, \ldots, x_{s-\sigma_{k s}}^{1}\right)-b_{s}\right]-c_{2} \\
& -\sum_{j=1}^{\infty} \sum_{i=n+(2 j-1) \tau_{0}}^{n+2 j \tau_{0}-1} \frac{1}{a_{i} h\left(x_{i-\tau_{1 i}}^{2}, x_{i-\tau_{2 i}}^{2}, \ldots, x_{i-\tau_{m i}}^{2}\right)} \\
& \cdot \sum_{s=n_{0}}^{i-1}\left[f\left(s, x_{s-\sigma_{1 s}}^{2}, x_{s-\sigma_{2 s}}^{2}, \ldots, x_{s-\sigma_{k s}}^{2}\right)-b_{s}\right]|\geq| c_{1} \\
& -c_{2} \mid-\sum_{j=1}^{\infty} \sum_{i=n+(2 j-1) \tau_{0}}^{n+2 j \tau_{0}-1} \frac{1}{\left|a_{i}\right|} \\
& \cdot \sum_{s=n_{0}}^{i-1}\left[\mid \frac{f\left(s, x_{s-\sigma_{1 s}}^{1}, x_{s-\sigma_{2 s}}^{1}, \ldots, x_{s-\sigma_{k s}}^{1}\right)}{h\left(x_{i-\tau_{1 i}}^{1}, x_{i-\tau_{2 i}}^{1}, \ldots, x_{i-\tau_{m i}}^{1}\right)}\right. \\
& -\frac{f\left(s, x_{s-\sigma_{1 s}}^{2}, x_{s-\sigma_{2 s}}^{2}, \ldots, x_{s-\sigma_{k s}}^{2}\right)}{h\left(x_{i-\tau_{1 i}}^{1}, x_{i-\tau_{2 i}}^{1}, \ldots, x_{i-\tau_{m i}}^{1}\right)} \mid \\
& +\mid \frac{f\left(s, x_{s-\sigma_{1 s}}^{2}, x_{s-\sigma_{2 s}}^{2}, \ldots, x_{s-\sigma_{k s}}^{2}\right)}{h\left(x_{i-\tau_{1 i}}^{1}, x_{i-\tau_{2 i}}^{1}, \ldots, x_{i-\tau_{m i}}^{1}\right)} \\
& -\frac{f\left(s, x_{s-\sigma_{1 s}}^{2}, x_{s-\sigma_{2 s}}^{2}, \ldots, x_{s-\sigma_{k s}}^{2}\right)}{h\left(x_{i-\tau_{1 i}}^{2}, x_{i-\tau_{2 i}}^{2}, \ldots, x_{i-\tau_{m i}}^{2}\right)}|+| b_{s} \mid
\end{aligned}
$$




$$
\begin{aligned}
& \left.\cdot\left|\frac{1}{h\left(x_{i-\tau_{1 i}}^{1}, x_{i-\tau_{2 i}}^{1}, \ldots, x_{i-\tau_{m i}}^{1}\right)}\right|\right] \\
& \left.-\frac{1}{h\left(x_{i-\tau_{1 i}}^{2}, x_{i-\tau_{2 i}}^{2}, \ldots, x_{i-\tau_{m i}}^{2}\right)} \mid\right] \geq\left|c_{1}-c_{2}\right| \\
& -\sum_{j=1}^{\infty} \sum_{i=n+(2 j-1) \tau_{0}}^{n+2 j \tau_{0}-1} \frac{1}{\left|a_{i}\right|} \sum_{s=n_{0}}^{i-1}\left[\frac{P_{s}}{m_{h}}\right. \\
& \cdot \max \left\{\left|x_{s-\sigma_{j s}}^{1}-x_{s-\sigma_{j s}}^{2}\right|: 1 \leq j \leq k\right\} \\
& +\frac{L_{h}}{m_{h}^{2}}\left(\left|f\left(s, x_{s-\sigma_{1 s}}^{2}, x_{s-\sigma_{2 s}}^{2}, \ldots, x_{s-\sigma_{k s}}^{2}\right)\right|+\left|b_{s}\right|\right) \\
& \left.\cdot \max \left\{\left|x_{i-\tau_{j i}}^{1}-x_{i-\tau_{j i}}^{2}\right|: 1 \leq j \leq m\right\}\right] \geq\left|c_{1}-c_{2}\right| \\
& -\frac{1}{m_{h}^{2}} \sum_{i=\max \left\{D_{1}, D_{2}\right\}}^{\infty} \frac{1}{\left|a_{i}\right|} \sum_{s=n_{0}}^{i-1}\left[m_{h} P_{s}+L_{h}\left(W_{s}+\left|b_{s}\right|\right)\right] \\
& \cdot|| x^{1}-x^{2}\left\|\geq\left|c_{1}-c_{2}\right|-\max \left\{\theta_{1}, \theta_{2}\right\}|| x^{1}-x^{2}\right\|,
\end{aligned}
$$

which yields that

$$
\left\|x^{1}-x^{2}\right\| \geq \frac{\left|c_{1}-c_{2}\right|}{1+\max \left\{\theta_{1}, \theta_{2}\right\}}>0,
$$

which ensures that $x^{1} \neq x^{2}$. This completes the proof.

Theorem 8. Assume that there exist constants $M, N, L_{h}$, and $m_{h}$ with $0<N<M$ and two nonnegative sequences $\left\{P_{n}\right\}_{n \in \mathbb{N}_{n_{0}}}$ and $\left\{W_{n}\right\}_{n \in \mathbb{N}_{n_{0}}}$ satisfying (15) (18):

$$
q_{n}=1, \text { eventually, }
$$

$$
\sum_{i=n_{0}+1}^{\infty} \frac{i}{\left|a_{i}\right|} \sum_{s=n_{0}}^{i-1} \max \left\{W_{s}, P_{s},\left|b_{s}\right|\right\}<+\infty
$$

Then, one has the following.

(a) For any $c \in(N, M)$, there exist $\theta \in(0,1)$ and $D \geq 1+n_{0}+\tau_{0}+|\beta|$ such that, for each $x_{0}=\left\{x_{0, n}\right\}_{n \in \mathbb{Z}_{\beta}} \in$ $A(N, M)$, the Mann iterative sequence with errors $\left\{x_{\eta}\right\}_{\eta \in \mathbb{N}_{0}}=$ $\left\{x_{\eta, n}\right\}_{(\eta, n) \in \mathbb{N}_{0} \times \mathbb{Z}_{\beta}}$ generated by the scheme

$$
\begin{aligned}
& x_{\eta+1, n} \\
& =\left\{\begin{array}{l}
\left(1-\alpha_{\eta}-\beta_{\eta}\right) x_{\eta, n}+\alpha_{\eta}\left\{c+\sum_{j=1}^{\infty} \sum_{i=n+j \tau_{0}}^{\infty} \frac{1}{a_{i} h\left(x_{\eta, i-\tau_{1 i}}, x_{\eta, i-\tau_{2 i}}, \ldots, x_{\eta, i-\tau_{m i}}\right)} \sum_{s=n_{0}}^{i-1}\left[f\left(s, x_{\eta, s-\sigma_{1 s}}, x_{\eta, s-\sigma_{2 s}}, \ldots, x_{\eta, s-\sigma_{k s}}\right)-b_{s}\right]\right\}+\beta_{\eta} \gamma_{\eta, n}, \quad n \geq D, \eta \geq 0, \\
\left(1-\alpha_{\eta}-\beta_{\eta}\right) x_{\eta, D}+\alpha_{\eta}\left\{c+\sum_{j=1}^{\infty} \sum_{i=D+j \tau_{0}}^{\infty} \frac{1}{a_{i} h\left(x_{\eta, i-\tau_{1 i}}, x_{\eta, i-\tau_{2 i}}, \ldots, x_{\eta, i-\tau_{m i}}\right)} \sum_{s=n_{0}}^{i-1}\left[f\left(s, x_{\eta, s-\sigma_{1 s}}, x_{\eta, s-\sigma_{2 s}}, \ldots, x_{\eta, s-\sigma_{k s}}\right)-b_{s}\right]\right\}+\beta_{\eta} \gamma_{\eta, D}, \quad \beta \leq n<D, \eta \geq 0,
\end{array}\right.
\end{aligned}
$$

converges to a bounded positive solution $x \in A(N, M)$ of (1) and has the error estimate (21), where $\left\{\gamma_{\eta}\right\}_{\eta \in \mathbb{N}_{0}}=$ $\left\{\gamma_{\eta, n}\right\}_{(\eta, n) \in \mathbb{N}_{0} \times \mathbb{Z}_{\beta}}$ is an arbitrary sequence in $A(N, M)$ and $\left\{\alpha_{\eta}\right\}_{\eta \in \mathbb{N}_{0}}$ and $\left\{\beta_{\eta}\right\}_{\eta \in \mathbb{N}_{0}}$ are any sequences in $[0,1]$ satisfying (22).

(b) Equation (1) has uncountably many bounded positive solutions in $A(N, M)$.

Proof. First, we show that (a) holds. Let $c \in(N, M)$. It follows from Lemma 2 and (69) that

$$
\sum_{j=0}^{\infty} \sum_{i=n_{0}+1+j \tau_{0}}^{\infty} \frac{1}{\left|a_{i}\right|} \sum_{s=n_{0}}^{i-1} \max \left\{W_{s}, P_{s},\left|b_{s}\right|\right\}<+\infty,
$$

which guarantees that there exist $\theta \in(0,1)$ and $D \geq 1+n_{0}+$ $\tau_{0}+|\beta|$ sufficiently large such that

$$
\begin{aligned}
& \theta=\frac{1}{m_{h}^{2}} \sum_{j=1}^{\infty} \sum_{i=D+j \tau_{0}}^{\infty} \frac{1}{\left|a_{i}\right|} \sum_{s=n_{0}}^{i-1}\left[m_{h} P_{s}+L_{h}\left(W_{s}+\left|b_{s}\right|\right)\right] ; \\
& \frac{1}{m_{h}} \sum_{j=1}^{\infty} \sum_{i=D+j \tau_{0}}^{\infty} \frac{1}{\left|a_{i}\right|} \sum_{s=n_{0}}^{i-1}\left(W_{s}+\left|b_{s}\right|\right) \\
& \quad<\min \{c-N, M-c\} .
\end{aligned}
$$

Define an operator $T: A(N, M) \rightarrow l_{\beta}^{\infty}$ by

$$
T x_{n}= \begin{cases}c+\sum_{j=1}^{\infty} \sum_{i=n+j \tau_{0}}^{\infty} \frac{1}{a_{i} h\left(x_{i-\tau_{1 i}}, x_{i-\tau_{2 i}}, \ldots, x_{i-\tau_{m i}}\right)} \sum_{s=n_{0}}^{i-1}\left[f\left(s, x_{s-\sigma_{1 s}}, x_{s-\sigma_{2 s}}, \ldots, x_{s-\sigma_{k s}}\right)-b_{s}\right], & n \geq D, \\ T x_{D}, & \beta \leq n<D\end{cases}
$$


for each $x=\left\{x_{n}\right\}_{n \in \mathbb{Z}_{\beta}} \in A(N, M)$. By virtue of (15) (18) and (72) (73), we get that, for every $x=\left\{x_{n}\right\}_{n \in \mathbb{Z}_{\beta}} \in A(N, M)$, $y=\left\{y_{n}\right\}_{n \in \mathbb{Z}_{\beta}} \in A(N, M)$, and $n \geq D$,

$$
\begin{aligned}
T x_{n} & =c+\sum_{j=1}^{\infty} \sum_{i=n+j \tau_{0}}^{\infty} \frac{1}{a_{i} h\left(x_{i-\tau_{1 i}}, x_{i-\tau_{2 i}}, \ldots, x_{i-\tau_{m i}}\right)} \\
\cdot & \sum_{s=n_{0}}^{i-1}\left[f\left(s, x_{s-\sigma_{1 s}}, x_{s-\sigma_{2 s}}, \ldots, x_{s-\sigma_{k s}}\right)-b_{s}\right] \geq c \\
& -\sum_{j=1}^{\infty} \sum_{i=n+j \tau_{0}}^{\infty} \frac{1}{\left|a_{i}\right| m_{h}} \\
& \cdot \sum_{s=n_{0}}^{i-1}\left[\left|f\left(s, x_{s-\sigma_{1 s}}, x_{s-\sigma_{2 s}}, \ldots, x_{s-\sigma_{k s}}\right)\right|+\left|b_{s}\right|\right] \geq c \\
& -\frac{1}{m_{h}} \sum_{j=1}^{\infty} \sum_{i=D+j \tau_{0}}^{\infty} \frac{1}{\left|a_{i}\right|} \sum_{s=n_{0}}^{i-1}\left(W_{s}+\left|b_{s}\right|\right) \geq c-\min \{c \\
& -N, M-c\} \geq N,
\end{aligned}
$$$$
T x_{n}=c+\sum_{j=1}^{\infty} \sum_{i=n+j \tau_{0}}^{\infty} \frac{1}{a_{i} h\left(x_{i-\tau_{1 i}}, x_{i-\tau_{2 i}}, \ldots, x_{i-\tau_{m i}}\right)}
$$$$
\cdot \sum_{s=n_{0}}^{i-1}\left[f\left(s, x_{s-\sigma_{1 s}}, x_{s-\sigma_{2 s}}, \ldots, x_{s-\sigma_{k s}}\right)-b_{s}\right] \leq c
$$$$
+\sum_{j=1}^{\infty} \sum_{i=n+j \tau_{0}}^{\infty} \frac{1}{\left|a_{i}\right| m_{h}}
$$$$
\cdot \sum_{s=n_{0}}^{i-1}\left[\left|f\left(s, x_{s-\sigma_{1 s}}, x_{s-\sigma_{2 s}}, \ldots, x_{s-\sigma_{k s}}\right)\right|+\left|b_{s}\right|\right] \leq c
$$$$
+\frac{1}{m_{h}} \sum_{j=1}^{\infty} \sum_{i=D+j \tau_{0}}^{\infty} \frac{1}{\left|a_{i}\right|} \sum_{s=n_{0}}^{i-1}\left(W_{s}+\left|b_{s}\right|\right) \leq c+\min \{c
$$$$
-N, M-c\} \leq M \text {, }
$$$$
\left|T x_{n}-T y_{n}\right|=\mid \sum_{j=1}^{\infty} \sum_{i=n+j \tau_{0}}^{\infty} \frac{1}{a_{i} h\left(x_{i-\tau_{1 i}}, x_{i-\tau_{2 i}}, \ldots, x_{i-\tau_{m i}}\right)}
$$$$
\cdot \sum_{s=n_{0}}^{i-1}\left[f\left(s, x_{s-\sigma_{1 s}}, x_{s-\sigma_{2 s}}, \ldots, x_{s-\sigma_{k s}}\right)-b_{s}\right]
$$$$
-\sum_{j=1}^{\infty} \sum_{i=n+j \tau_{0}}^{\infty} \frac{1}{a_{i} h\left(y_{i-\tau_{1 i}}, y_{i-\tau_{2 i}}, \ldots, y_{i-\tau_{m i}}\right)}
$$$$
\cdot \sum_{s=n_{0}}^{i-1}\left[f\left(s, y_{s-\sigma_{1 s}}, y_{s-\sigma_{2 s}}, \ldots, y_{s-\sigma_{k s}}\right)-b_{s}\right]
$$$$
\leq \sum_{j=1}^{\infty} \sum_{i=n+j \tau_{0}}^{\infty} \frac{1}{\left|a_{i}\right|} \sum_{s=n_{0}}^{i-1}\left[\mid \frac{f\left(s, x_{s-\sigma_{1 s}}, x_{s-\sigma_{2 s}}, \ldots, x_{s-\sigma_{k s}}\right)}{h\left(x_{i-\tau_{1 i}}, x_{i-\tau_{2 i}}, \ldots, x_{i-\tau_{m i}}\right)}\right.
$$

$$
\begin{aligned}
& -\frac{f\left(s, y_{s-\sigma_{1 s}}, y_{s-\sigma_{2 s}}, \ldots, y_{s-\sigma_{k s}}\right)}{h\left(x_{i-\tau_{1 i}}, x_{i-\tau_{2 i}}, \ldots, x_{i-\tau_{m i}}\right)} \mid \\
& +\mid \frac{f\left(s, y_{s-\sigma_{1 s}}, y_{s-\sigma_{2 s}}, \ldots, y_{s-\sigma_{k s}}\right)}{h\left(x_{i-\tau_{1 i}}, x_{i-\tau_{2 i}}, \ldots, x_{i-\tau_{m i}}\right)} \\
& -\frac{f\left(s, y_{s-\sigma_{1 s}}, y_{s-\sigma_{2 s}}, \ldots, y_{s-\sigma_{k s}}\right)}{h\left(y_{i-\tau_{1 i}}, y_{i-\tau_{2 i}}, \ldots, y_{i-\tau_{m i}}\right)}|+| b_{s} \mid
\end{aligned}
$$$$
\cdot \mid \frac{1}{h\left(x_{i-\tau_{1 i}}, x_{i-\tau_{2 i}}, \ldots, x_{i-\tau_{m i}}\right)}
$$$$
\left.-\frac{1}{h\left(y_{i-\tau_{1 i}}, y_{i-\tau_{2 i}}, \ldots, y_{i-\tau_{m i}}\right)} \mid\right] \leq \sum_{j=1}^{\infty} \sum_{i=n+j \tau_{0}}^{\infty} \frac{1}{\left|a_{i}\right|}
$$$$
\cdot \sum_{s=n_{0}}^{i-1}\left[\frac{P_{s}}{m_{h}} \max \left\{\left|x_{s-\sigma_{j s}}-y_{s-\sigma_{j s}}\right|: 1 \leq j \leq k\right\}\right.
$$$$
+\frac{L_{h}}{m_{h}^{2}}\left(\left|f\left(s, y_{s-\sigma_{1 s}}, y_{s-\sigma_{2 s}}, \ldots, y_{s-\sigma_{k s}}\right)\right|+\left|b_{s}\right|\right)
$$$$
\left.\cdot \max \left\{\left|x_{i-\tau_{j i}}-y_{i-\tau_{j i}}\right|: 1 \leq j \leq m\right\}\right] \leq \frac{1}{m_{h}^{2}}
$$$$
\cdot \sum_{j=1}^{\infty} \sum_{i=n+j \tau_{0}}^{\infty} \frac{1}{\left|a_{i}\right|} \sum_{s=n_{0}}^{i-1}\left[m_{h} P_{s}+L_{h}\left(W_{s}+\left|b_{s}\right|\right)\right]\|x-y\|
$$$$
\leq \theta\|x-y\| \text {, }
$$

which yield (26); that is, $T$ is a contraction operator in $A(N, M)$ and $T$ has a unique fixed point $x=\left\{x_{n}\right\}_{n \in \mathbb{Z}_{\beta}} \in$ $A(N, M)$ with

$$
\begin{aligned}
x_{n}=c & +\sum_{j=1}^{\infty} \sum_{i=n+j \tau_{0}}^{\infty} \frac{1}{a_{i} h\left(x_{i-\tau_{1 i}}, x_{i-\tau_{2 i}}, \ldots, x_{i-\tau_{m i}}\right)} \\
& \cdot \sum_{s=n_{0}}^{i-1}\left[f\left(s, x_{s-\sigma_{1 s}}, x_{s-\sigma_{2 s}}, \ldots, x_{s-\sigma_{k s}}\right)-b_{s}\right],
\end{aligned}
$$

which yields that

$$
\begin{array}{r}
x_{n}-x_{n-\tau_{0}}=-\sum_{i=n}^{\infty} \frac{1}{a_{i} h\left(x_{i-\tau_{1 i}}, x_{i-\tau_{2 i}}, \ldots, x_{i-\tau_{m i}}\right)} \\
\cdot \sum_{s=n_{0}}^{i-1}\left[f\left(s, x_{s-\sigma_{1 s}}, x_{s-\sigma_{2 s}}, \ldots, x_{s-\sigma_{k s}}\right)-b_{s}\right],
\end{array}
$$

$\forall n \geq D$ 


$$
\begin{gathered}
\Delta\left(x_{n}-x_{n-\tau_{0}}\right)=-\frac{1}{a_{n} h\left(x_{n-\tau_{1 n}}, x_{n-\tau_{2 n}}, \ldots, x_{n-\tau_{m n}}\right)} \\
\cdot \sum_{s=n_{0}}^{n-1}\left[f\left(s, x_{s-\sigma_{1 s}}, x_{s-\sigma_{2 s}}, \ldots, x_{s-\sigma_{k s}}\right)-b_{s}\right],
\end{gathered}
$$

$\forall n \geq D$,

which implies that

$$
\begin{aligned}
& \Delta\left(a_{n} h\left(x_{n-\tau_{1 n}}, x_{n-\tau_{2 n}}, \ldots, x_{n-\tau_{m n}}\right) \Delta\left(x_{n}-x_{n-\tau_{0}}\right)\right) \\
& \quad=-f\left(n, x_{n-\sigma_{1 n}}, x_{n-\sigma_{2 n}}, \ldots, x_{n-\sigma_{k n}}\right)+b_{n}, \quad \forall n \geq D,
\end{aligned}
$$

which means that $x=\left\{x_{n}\right\}_{n \in \mathbb{Z}_{\beta}}$ is a bounded positive solution of (1) in $A(N, M)$.

It follows from (20), (26), and (73) that for any $\eta \geq 0$ and $n \geq D$

$$
\begin{aligned}
& \left|x_{\eta+1, n}-x_{n}\right|=\mid\left(1-\alpha_{\eta}-\beta_{\eta}\right) x_{\eta, n}+\alpha_{\eta}\{c \\
& +\sum_{j=1}^{\infty} \sum_{i=n+j \tau_{0}}^{\infty} \frac{1}{a_{i} h\left(x_{\eta, i-\tau_{1 i}}, x_{\eta, i-\tau_{2 i}}, \ldots, x_{\eta, i-\tau_{m i}}\right)} \\
& \left.\quad \sum_{s=n_{0}}^{i-1}\left[f\left(s, x_{\eta, s-\sigma_{1 s}}, x_{\eta, s-\sigma_{2 s}}, \ldots, x_{\eta, s-\sigma_{k s}}\right)-b_{s}\right]\right\} \\
& +\beta_{\eta} \gamma_{\eta, n}-x_{n}\left|\leq\left(1-\alpha_{\eta}-\beta_{\eta}\right)\right| x_{\eta, n}-x_{n} \mid \\
& +\alpha_{\eta}\left|T x_{\eta, n}-T x_{n}\right|+\beta_{\eta}\left|\gamma_{\eta, n}-x_{n}\right| \leq\left(1-\alpha_{\eta}-\beta_{\eta}\right) \\
& \cdot\left\|x_{\eta}-x\right\|+\alpha_{\eta} \theta\left\|x_{\eta}-x\right\|+2 M \beta_{\eta} \leq(1-(1-\theta) \\
& \left.\cdot \alpha_{\eta}\right)\left\|x_{\eta}-x\right\|+2 M \beta_{\eta},
\end{aligned}
$$

which implies that

$$
\left\|x_{\eta+1}-x\right\| \leq\left(1-(1-\theta) \alpha_{\eta}\right)\left\|x_{\eta}-x\right\|+2 M \beta_{\eta},
$$

$$
\eta \geq 0 \text {. }
$$

That is, (21) holds. Thus, Lemma 1 and (21) (22) ensure that $\lim _{\eta \rightarrow \infty} x_{\eta}=x$.

Next, we show that (b) holds. Let $c_{1}, c_{2} \in(N, M)$ with $c_{1} \neq c_{2}$. As in the proof of (a), we deduce that for each $l \in$ $\{1,2\}$ there exist constants $\theta_{l} \in(0,1), D_{l}>1+n_{0}+\tau_{0}+|\beta|$, and an operator $T_{l}: A(N, M) \rightarrow A(N, M)$ satisfying (26) and (72) (73), where $\theta, D$, and $T$ are replaced by $\theta_{l}, D_{l}$, and $T_{l}$, respectively, and the contraction operator $T_{l}$ has a unique fixed point $x^{l}=\left\{x_{n}^{l}\right\}_{n \in \mathbb{Z}_{\beta}} \in A(N, M)$, which is also a bounded positive solution of (1); that is,

$$
x_{n}^{l}=c_{l}+\sum_{j=1}^{\infty} \sum_{i=n+j \tau_{0}}^{\infty} \frac{1}{a_{i} h\left(x_{i-\tau_{1 i}}^{l}, x_{i-\tau_{2 i}}^{l}, \ldots, x_{i-\tau_{m i}}^{l}\right)}
$$

$$
\cdot \sum_{s=n_{0}}^{i-1}\left[f\left(s, x_{s-\sigma_{1 s}}^{l}, x_{s-\sigma_{2 s}}^{l}, \ldots, x_{s-\sigma_{k s}}^{l}\right)-b_{s}\right]
$$

$\forall n \geq D_{l}$,

which together with (15) (18), (26), and (72) (73) gives that for each $n \geq \max \left\{D_{1}, D_{2}\right\}$

$$
\begin{aligned}
& \left|x_{n}^{1}-x_{n}^{2}\right|=\mid c_{1} \\
& +\sum_{j=1}^{\infty} \sum_{i=n+j \tau_{0}}^{\infty} \frac{1}{a_{i} h\left(x_{i-\tau_{1 i}}^{1}, x_{i-\tau_{2 i}}^{1}, \ldots, x_{i-\tau_{m i}}^{1}\right)} \\
& \cdot \sum_{s=n_{0}}^{i-1}\left[f\left(s, x_{s-\sigma_{1 s}}^{1}, x_{s-\sigma_{2 s}}^{1}, \ldots, x_{s-\sigma_{k s}}^{1}\right)-b_{s}\right]-c_{2} \\
& -\sum_{j=1}^{\infty} \sum_{i=n+j \tau_{0}}^{\infty} \frac{1}{a_{i} h\left(x_{i-\tau_{1 i}}^{2}, x_{i-\tau_{2 i}}^{2}, \ldots, x_{i-\tau_{m i}}^{2}\right)} \\
& \cdot \sum_{s=n_{0}}^{i-1}\left[f\left(s, x_{s-\sigma_{1 s}}^{2}, x_{s-\sigma_{2 s}}^{2}, \ldots, x_{s-\sigma_{k s}}^{2}\right)-b_{s}\right]|\geq| c_{1} \\
& -c_{2} \mid-\sum_{j=1}^{\infty} \sum_{i=n+j \tau_{0}}^{\infty} \frac{1}{\left|a_{i}\right|} \\
& \cdot \sum_{s=n_{0}}^{i-1}\left[\mid \frac{f\left(s, x_{s-\sigma_{1 s}}^{1}, x_{s-\sigma_{2 s}}^{1}, \ldots, x_{s-\sigma_{k s}}^{1}\right)}{h\left(x_{i-\tau_{1 i}}^{1}, x_{i-\tau_{2 i}}^{1}, \ldots, x_{i-\tau_{m i}}^{1}\right)}\right. \\
& -\frac{f\left(s, x_{s-\sigma_{1 s}}^{2}, x_{s-\sigma_{2 s}}^{2}, \ldots, x_{s-\sigma_{k s}}^{2}\right)}{h\left(x_{i-\tau_{1 i}}^{1}, x_{i-\tau_{2 i}}^{1}, \ldots, x_{i-\tau_{m i}}^{1}\right)} \mid \\
& +\mid \frac{f\left(s, x_{s-\sigma_{1 s}}^{2}, x_{s-\sigma_{2 s}}^{2}, \ldots, x_{s-\sigma_{k s}}^{2}\right)}{h\left(x_{i-\tau_{1 i}}^{1}, x_{i-\tau_{2 i}}^{1}, \ldots, x_{i-\tau_{m i}}^{1}\right)} \\
& -\frac{f\left(s, x_{s-\sigma_{1 s}}^{2}, x_{s-\sigma_{2 s}}^{2}, \ldots, x_{s-\sigma_{k s}}^{2}\right)}{h\left(x_{i-\tau_{1 i}}^{2}, x_{i-\tau_{2 i}}^{2}, \ldots, x_{i-\tau_{m i}}^{2}\right)}|+| b_{s} \mid \\
& \cdot \mid \frac{1}{h\left(x_{i-\tau_{1 i}}^{1}, x_{i-\tau_{2 i}}^{1}, \ldots, x_{i-\tau_{m i}}^{1}\right)} \\
& \left.-\frac{1}{h\left(x_{i-\tau_{1 i}}^{2}, x_{i-\tau_{2 i}}^{2}, \ldots, x_{i-\tau_{m i}}^{2}\right)} \mid\right] \geq\left|c_{1}-c_{2}\right|
\end{aligned}
$$




$$
\begin{aligned}
& -\sum_{j=1}^{\infty} \sum_{i=n+j \tau_{0}}^{\infty} \frac{1}{\left|a_{i}\right|} \sum_{s=n_{0}}^{i-1}\left[\frac{P_{s}}{m_{h}}\right. \\
& \cdot \max \left\{\left|x_{s-\sigma_{j s}}^{1}-x_{s-\sigma_{j s}}^{2}\right|: 1 \leq j \leq k\right\} \\
& +\frac{L_{h}}{m_{h}^{2}}\left(\left|f\left(s, x_{s-\sigma_{1 s}}^{2}, x_{s-\sigma_{2 s}}^{2}, \ldots, x_{s-\sigma_{k s}}^{2}\right)\right|+\left|b_{s}\right|\right) \\
& \left.\cdot \max \left\{\left|x_{i-\tau_{j i}}^{1}-x_{i-\tau_{j i} \mid}^{2}\right|: 1 \leq j \leq m\right\}\right] \geq\left|c_{1}-c_{2}\right| \\
& -\frac{1}{m_{h}^{2}} \sum_{j=1}^{\infty} \sum_{i=\max \left\{D_{1}, D_{2}\right\}+j \tau_{0}}^{\infty} \frac{1}{\left|a_{i}\right|} \sum_{s=n_{0}}^{i-1}\left[m_{h} P_{s}+L_{h}\left(W_{s}\right.\right. \\
& \left.\left.+\left|b_{s}\right|\right)\right]\left\|x^{1}-x^{2}\right\| \geq\left|c_{1}-c_{2}\right|-\max \left\{\theta_{1}, \theta_{2}\right\} \| x^{1} \\
& -x^{2} \|,
\end{aligned}
$$

which yields that

$$
\left\|x^{1}-x^{2}\right\| \geq \frac{\left|c_{1}-c_{2}\right|}{1+\max \left\{\theta_{1}, \theta_{2}\right\}}>0 ;
$$

that is, $x^{1} \neq x^{2}$. This completes the proof.

Remark 9. Theorems 3 8 extend and unify Theorem 1 in [3] and Theorems $2.1 \sim 2.7$ in [12], respectively.

\section{Examples}

In what follows, we construct six examples to demonstrate that Theorems $3 \sim 8$ extend properly the corresponding results in $[3,12]$, respectively. Note that none of the known results can be applied to the examples.

Example 1. Consider the second-order nonlinear neutral delay difference equation with forcing term

$$
\begin{aligned}
& \Delta\left((-1)^{n-1} \sqrt{n^{3}-n^{2}+1}\left(x_{n^{2}+n-1}^{2}+x_{n(n+1) / 2}\right)\right. \\
& \left.\cdot \Delta\left(x_{n}-\frac{n+1}{2 n+3} x_{n-\tau_{0}}\right)\right)+\frac{n^{2} x_{2 n^{2}-n}+x_{n-5}^{2}}{n^{4}} \\
& =\frac{\sin (n-\sqrt{n-\sqrt{n}})}{n^{2}}, \quad n \geq 1,
\end{aligned}
$$

where $\tau_{0} \in \mathbb{N}$ is fixed. Let $n_{0}=1, m=k=2, q=1 / 2, M=6$, $N=2, \beta=\min \left\{1-\tau_{0},-4\right\}$,

$$
\begin{aligned}
& a_{n}=(-1)^{n-1} \sqrt{n^{3}-n^{2}+1}, \\
& q_{n}=\frac{n+1}{2 n+3},
\end{aligned}
$$

$$
\begin{aligned}
b_{n} & =\frac{\sin (n-\sqrt{n-\sqrt{n}})}{n^{2}}, \\
\tau_{1 n} & =-n^{2}+1, \\
\tau_{2 n} & =\frac{n(1-n)}{2}, \\
\sigma_{1 n} & =2 n(1-n), \\
\sigma_{2 n} & =5, \\
f(n, u, v) & =\frac{n^{2} u+v^{2}}{n^{4}}, \\
h(u, v) & =u^{2}+v, \\
P_{n} & =\frac{n^{2}+2 M}{n^{4}}, \\
W_{n} & =\frac{n^{2} M+M^{2}}{n^{4}}, \\
L_{h} & =2 M+1, \\
m_{h} & =N^{2}+N,
\end{aligned}
$$

$n \in \mathbb{N}_{n_{0}}, u, v \in \mathbb{R}$.

It is easy to show that (13) (18) are satisfied. Note that

$$
\begin{aligned}
& \sum_{i=n_{0}+1}^{\infty} \frac{1}{\left|a_{i}\right|} \sum_{s=n_{0}}^{i-1} \max \left\{W_{s}, P_{s},\left|b_{s}\right|\right\}=\sum_{i=n_{0}+1}^{\infty} \frac{1}{\sqrt{i^{3}-i^{2}+1}} \\
& \cdot \sum_{s=n_{0}}^{i-1} \max \left\{\frac{s^{2} M+M^{2}}{s^{4}}, \frac{s^{2}+2 M}{s^{4}},\right. \\
& \left.\frac{|\sin (s-\sqrt{s-\sqrt{s}})|}{s^{2}}\right\} \leq 2 M(1+M) \\
& \cdot \sum_{i=n_{0}+1}^{\infty} \frac{1}{\sqrt{i^{3}-i^{2}+1}}<+\infty
\end{aligned}
$$

that is, (19) holds. It follows from Theorem 3 that (83) has uncountably many bounded positive solutions in $A(N, M)$. On the other hand, for each $c \in(N,(1-q) M)$, there exist $\theta \in(0,1)$ and $T \geq n_{0}+\tau_{0}+|\beta|$ such that the Mann iterative sequence with errors $\left\{x_{m}\right\}_{m \geq 0}$ generated by (20) converges to a bounded positive solution $x \in A(N, M)$ of (83) and has the error estimate (21), where $\left\{\gamma_{\eta}\right\}_{\eta \geq 0}$ is an arbitrary sequence in $A(N, M)$ and $\left\{\alpha_{\eta}\right\}_{\eta \geq 0}$ and $\left\{\beta_{\eta}\right\}_{\eta \geq 0}$ are any sequences in $[0,1]$ satisfying (22). 
Example 2. Consider the second-order nonlinear neutral delay difference equation with forcing term

$$
\begin{aligned}
& \Delta\left((-1)^{n-1} n^{5}\left(\frac{x_{n^{3}}+6 x_{4 n-3}^{2}}{1+x_{n^{3}}^{2}}\right)\right. \\
& \left.\cdot \Delta\left(x_{n}+\frac{4 n^{5}}{5 n^{5}+3} x_{n-\tau_{0}}\right)\right) \\
& +\frac{\left(n^{2}+1\right) x_{2^{n}+n-1}^{2}-n^{3} x_{n+6}}{n^{7}+2 n^{5}+1} \\
& =\frac{(-1)^{n-1} n^{2}+5 n-1}{n^{6}+20 n^{5}+3 n^{4}+n+5}, \quad n \geq 2,
\end{aligned}
$$

where $\tau_{0} \in \mathbb{N}$ is fixed. Let $n_{0}=m=k=2, q=-4 / 5, M=8$, $N=1, \beta=2-\tau_{0}$,

$$
\begin{aligned}
a_{n} & =(-1)^{n-1} n^{5}, \\
q_{n} & =-\frac{4 n^{5}}{5 n^{5}+3}, \\
b_{n} & =\frac{(-1)^{n-1} n^{2}+5 n-1}{n^{6}+20 n^{5}+3 n^{4}+n+5}, \\
\tau_{1 n} & =n-n^{3}, \\
\tau_{2 n} & =-3 n+3, \\
\sigma_{1 n} & =-2^{n}+1, \\
\sigma_{2 n} & =-6, \\
f(n, u, v) & =\frac{\left(n^{2}+1\right) u^{2}-n^{3} v}{n^{7}+2 n^{5}+1}, \\
h(u, v) & =\frac{v+6 u^{2}}{1+v^{2}}, \\
P_{n} & =\frac{4 M}{n^{5}}+\frac{1}{n^{4}}, \\
W_{n} & =\frac{2 M^{2}}{n^{5}}+\frac{M}{n^{4}}, \\
L_{h} & =\frac{7+19 M^{2}}{\left(1+N^{2}\right)^{2}}, \\
m_{h} & =\frac{N+6 N^{2}}{1+M^{2}},
\end{aligned}
$$

$$
n \in \mathbb{N}_{n_{0}}, u, v \in \mathbb{R} .
$$

It is clear that (15) (18), (34), and (35) hold. Notice that

$$
\begin{gathered}
\sum_{i=n_{0}+1}^{\infty} \frac{1}{\left|a_{i}\right|} \sum_{s=n_{0}}^{i-1} \max \left\{W_{s}, P_{s},\left|b_{s}\right|\right\}=\sum_{i=n_{0}+1}^{\infty} \frac{1}{i^{5}} \\
\cdot \sum_{s=n_{0}}^{i-1} \max \left\{\frac{4 M}{s^{5}}+\frac{1}{s^{4}}, \frac{M^{2}}{s^{5}}\right.
\end{gathered}
$$

$$
\begin{aligned}
& \left.+\frac{M}{s^{4}}, \frac{\left|(-1)^{s-1} s^{2}+5 s-1\right|}{s^{6}+20 s^{5}+3 s^{4}+s+5}\right\} \leq \sum_{i=n_{0}+1}^{\infty} \frac{1}{i^{5}} \sum_{s=n_{0}}^{i-1} \frac{2 M^{2}}{s^{4}} \\
& \leq 4 M^{2} \sum_{i=n_{0}+1}^{\infty} \frac{1}{i^{5}}<+\infty,
\end{aligned}
$$

which implies (19). It follows from Theorem 4 that (86) has uncountably bounded positive solutions in $A(N, M)$. On the other hand, for any $c \in(N-q M, M)$, there exist $\theta \in$ $(0,1)$ and $T \geq n_{0}+\tau_{0}+|\beta|$ such that the Mann iterative sequence with errors $\left\{x_{m}\right\}_{m \geq 0}$ generated by (20) converges to a bounded positive solution $x \in A(N, M)$ of (86) and has the error estimate (21), where $\left\{\gamma_{\eta}\right\}_{\eta \geq 0}$ is an arbitrary sequence in $A(N, M)$ and $\left\{\alpha_{\eta}\right\}_{\eta \geq 0}$ and $\left\{\beta_{\eta}\right\}_{\eta \geq 0}$ are any sequences in $[0,1]$ satisfying (22).

Example 3. Consider the second-order nonlinear neutral delay difference equation with forcing term

$$
\begin{aligned}
& \Delta\left((-1)^{n(n+1) / 2}\left(n^{6}+3^{n}+2\right)\left(\frac{3+x_{n^{2}+(-1)^{n} n}^{2 / 3}}{1+x_{5 n+1}^{2}}\right)\right. \\
& \left.\cdot \Delta\left(x_{n}-\frac{5^{n+1}+4}{5^{n}+1} x_{n-\tau_{0}}\right)\right)+\frac{n x_{n^{2}+n}+x_{3 n^{2}+n-2}^{2}}{n^{5}+2 n} \\
& =\frac{(-1)^{n} n+\arctan n}{n^{3}+4 n^{2}+2}, \quad n \geq 1,
\end{aligned}
$$

where $\tau_{0} \in \mathbb{N}$ is fixed. Let $n_{0}=1, m=k=2, \underline{q}=2, \bar{q}=5$, $M=9, N=1, \beta=1-\tau_{0}$,

$$
\begin{aligned}
a_{n} & =(-1)^{n(n+1) / 2}\left(n^{6}+3^{n}+2\right), \\
q_{n} & =\frac{5^{n+1}+4}{5^{n}+1}, \\
b_{n} & =\frac{(-1)^{n} n+\arctan n}{n^{3}+4 n^{2}+2}, \\
\tau_{1 n} & =-n^{2}-(-1)^{n} n+n, \\
\tau_{2 n} & =-4 n-1, \\
\sigma_{1 n} & =-n^{2}, \\
\sigma_{2 n} & =-3 n^{2}+2, \\
(n, u, v) & =\frac{n u+v^{2}}{n^{5}+2 n}, \\
h(u, v) & =\frac{3+u^{2 / 3}}{1+v^{2}},
\end{aligned}
$$$$
f(n, u, v)=\frac{n u+v^{2}}{n^{5}+2 n}
$$ 


$$
\begin{aligned}
P_{n} & =\frac{2 M+n}{n^{5}+2 n}, \\
W_{n} & =\frac{n M+M^{2}}{n^{5}+2 n}, \\
L_{h} & =6 M^{3} \\
m_{h} & =\frac{3+N^{2 / 3}}{1+M^{2}},
\end{aligned}
$$$$
n \in \mathbb{N}_{n_{0}}, u, v \in \mathbb{R}
$$

It is easy to show that (15) (18), (38), and (39) are satisfied. Note that

$$
\begin{aligned}
& \sum_{i=n_{0}+1}^{\infty} \frac{1}{\left|a_{i}\right|} \sum_{s=n_{0}}^{i-1} \max \left\{W_{s}, P_{s},\left|b_{s}\right|\right\}=\sum_{i=n_{0}+1}^{\infty} \frac{1}{i^{6}+3^{i}+2} \\
& \cdot \sum_{s=n_{0}}^{i-1} \max \left\{\frac{s M+M^{2}}{s^{5}+2 s}, \frac{2 M+s}{s^{5}+2 s},\right. \\
& \left.\frac{\left|(-1)^{s} s+\arctan s\right|}{s^{3}+4 s^{2}+2}\right\} \leq \sum_{i=n_{0}+1}^{\infty} \frac{1}{3^{i}} \sum_{s=n_{0}}^{i-1} \frac{M+M^{2}}{s^{2}} \\
& \leq \frac{M+M^{2}}{3}<+\infty,
\end{aligned}
$$

which means (19). It follows from Theorem 5 that (89) has uncountably bounded positive solutions in $A(N, M)$. On the other hand, for any $c \in(\bar{q} N,(q-1) M)$, there exist $\theta \in$ $(0,1)$ and $T \geq n_{0}+\tau_{0}+|\beta|$ such that the Mann iterative sequence with errors $\left\{x_{m}\right\}_{m \geq 0}$ generated by (40) converges to a bounded positive solution $x \in A(N, M)$ of (89) and has the error estimate (21), where $\left\{\gamma_{\eta}\right\}_{\eta \geq 0}$ is an arbitrary sequence in $A(N, M)$ and $\left\{\alpha_{\eta}\right\}_{\eta \geq 0}$ and $\left\{\beta_{\eta}\right\}_{\eta \geq 0}$ are any sequences in $[0,1]$ satisfying (22).

Example 4. Consider the second-order nonlinear neutral delay difference equation with forcing term

$$
\begin{aligned}
& \Delta\left(n^{3}\left(2+\cos \left(n^{2}-1\right)\right)\left(\frac{x_{n^{3}+n-1}^{2}+x_{n^{3}+n-1}}{2+x_{n-3}^{2}}\right)\right. \\
& \left.\cdot \Delta\left(x_{n}+\frac{n^{3}+3 \sqrt{n}+7}{n^{3}+\sqrt{n}+1} x_{n-\tau_{0}}\right)\right) \\
& +\frac{n^{2} x_{9 n+3}+(n-1) x_{2 n-8}^{3}}{n^{4}+x_{2 n-8}^{2}}=\frac{(-1)^{n(n+1) / 2} n^{2}+n}{n^{4}+n^{3}+1}, \\
& \quad n \geq 1,
\end{aligned}
$$

where $\tau_{0} \in \mathbb{N}$ is fixed. Let $n_{0}=1, m=k=2, \bar{q}=-3, \underline{q}=-7$, $M=21, N=1, \beta=\min \left\{1-\tau_{0},-6\right\}$,

$$
\begin{aligned}
& a_{n}=n^{3}\left(2+\cos \left(n^{2}-1\right)\right), \\
& q_{n}=-\frac{n^{3}+3 \sqrt{n}+7}{n^{3}+\sqrt{n}+1},
\end{aligned}
$$

$$
\begin{aligned}
b_{n} & =\frac{(-1)^{n(n+1) / 2} n^{2}+n}{n^{4}+n^{3}+1}, \\
\tau_{1 n} & =3 \\
\tau_{2 n} & =-n^{3}+1, \\
\sigma_{1 n} & =-8 n-3, \\
\sigma_{2 n} & =-n+8 \\
f(n, u, v) & =\frac{n^{2} u+(n-1) v^{3}}{n^{4}+v^{2}}, \\
h(u, v) & =\frac{v^{2}+v}{2+u^{2}} \\
P_{n} & =\frac{7 M^{4}}{n^{2}} \\
m_{h} & =\frac{N^{2}+N}{2+M^{2}}, \\
W_{n} & =\frac{2 M^{3}}{n^{2}} \\
& =\frac{2+4 M+3 M^{2}+4 M^{3}}{\left(2+N^{2}\right)^{2}} \\
& \\
L_{h} & \\
w^{2} &
\end{aligned}
$$$$
n \in \mathbb{N}_{n_{0}}, u, v \in \mathbb{R}
$$

It is easy to show that (15) (18), (51), and (52) are satisfied. Note that

$$
\begin{aligned}
& \sum_{i=n_{0}+1}^{\infty} \frac{1}{\left|a_{i}\right|} \sum_{s=n_{0}}^{i-1} \max \left\{W_{s}, P_{s},\left|b_{s}\right|\right\} \\
& \quad=\sum_{i=n_{0}+1}^{\infty} \frac{1}{i^{3}\left(2+\cos \left(i^{2}-1\right)\right)} \\
& \quad \cdot \sum_{s=n_{0}}^{i-1} \max \left\{\frac{2 M^{3}}{s^{2}}, \frac{7 M^{4}}{s^{2}},\left|\frac{(-1)^{s(s+1) / 2} s^{2}+s}{s^{4}+s^{3}+1}\right|\right\} \\
& \quad \leq 14 M^{4} \sum_{i=n_{0}+1}^{\infty} \frac{1}{i^{3}}<+\infty ;
\end{aligned}
$$

that is, (19) holds. It follows from Theorem 6 that (92) has uncountably bounded positive solutions in $A(N, M)$. On the other hand, for any $c \in(\bar{q} M, \underline{q} N-\underline{q} M / \bar{q})$, there exist $\theta \in$ $(0,1)$ and $T \geq n_{0}+\tau_{0}+|\beta|$ such that the Mann iterative sequence with errors $\left\{x_{m}\right\}_{m \geq 0}$ generated by (40) converges to a bounded positive solution $x \in A(N, M)$ of (92) and has the error estimate (21), where $\left\{\gamma_{\eta}\right\}_{\eta \geq 0}$ is an arbitrary sequence in $A(N, M)$ and $\left\{\alpha_{\eta}\right\}_{\eta \geq 0}$ and $\left\{\beta_{\eta}\right\}_{\eta \geq 0}$ are any sequences in $[0,1]$ satisfying (22). 
Example 5. Consider the second-order nonlinear neutral delay difference equation with forcing term

$$
\begin{aligned}
& \Delta\left((-1)^{n-1} n^{2} \ln ^{3}(n+1)\left(\frac{2 x_{n(n+1) / 2}+x_{n+5}^{2}}{1+x_{n+5}^{2}}\right)\right. \\
& \left.\cdot \Delta\left(x_{n}+x_{n-\tau_{0}}\right)\right)+\frac{n^{2} x_{n+3} x_{2 n-5}+n x_{n+3}^{2}+x_{2 n-5}}{n^{5}+\left|x_{n+3}\right|} \\
& =\frac{n^{2}-2 n+2}{n^{5}+4 n^{3}+1}, \quad n \geq 1,
\end{aligned}
$$

where $\tau_{0} \in \mathbb{N}$ is fixed. Let $n_{0}=1, m=k=2, M=6, N=2$, $\beta=\min \left\{1-\tau_{0},-3\right\}$,

$$
\begin{aligned}
& a_{n}=(-1)^{n-1} n^{2} \ln ^{3}(n+1) \text {, } \\
& q_{n}=-1 \text {, } \\
& b_{n}=\frac{n^{2}-2 n+2}{n^{5}+4 n^{3}+1}, \\
& \tau_{1 n}=-\frac{n(n-1)}{2} \text {, } \\
& \tau_{2 n}=-5 \text {, } \\
& \sigma_{1 n}=5-n \text {, } \\
& \sigma_{2 n}=-3 \text {, } \\
& f(n, u, v)=\frac{n^{2} u v+n u^{2}+v}{n^{5}+|u|}, \\
& h(u, v)=\frac{2 u+v^{2}}{1+v^{2}}, \\
& P_{n}=\frac{4 M^{2}}{n^{3}}, \\
& W_{n}=\frac{3 M^{2}}{n^{3}}, \\
& L_{h}=\frac{7 M^{2}}{\left(1+N^{2}\right)^{2}} \text {, } \\
& m_{h}=\frac{2 N+N^{2}}{1+M^{2}},
\end{aligned}
$$$$
n \in \mathbb{N}_{n_{0}}, u, v \in \mathbb{R}
$$

It is easy to show that (15) (18) and (55) hold. Note that

$$
\begin{aligned}
& \sum_{i=n_{0}+1}^{\infty} \frac{1}{\left|a_{i}\right|} \sum_{s=n_{0}}^{i-1} \max \left\{W_{s}, P_{s},\left|b_{s}\right|\right\}=\sum_{i=n_{0}+1}^{\infty} \frac{1}{i^{2} \ln ^{3}(i+1)} \\
& \quad \cdot \sum_{s=n_{0}}^{i-1} \max \left\{\frac{3 M^{2}}{s^{3}}, \frac{4 M^{2}}{s^{3}}, \frac{s^{2}-2 s+2}{s^{5}+4 s^{3}+1}\right\}
\end{aligned}
$$

$$
\begin{aligned}
& =\sum_{i=n_{0}+1}^{\infty} \frac{1}{i^{2} \ln ^{3}(i+1)} \sum_{s=n_{0}}^{i-1} \frac{4 M^{2}}{s^{3}} \\
& \leq 8 M^{2} \sum_{i=n_{0}+1}^{\infty} \frac{1}{i^{2} \ln ^{3}(i+1)}<+\infty ;
\end{aligned}
$$

that is, (19) holds. It follows from Theorem 7 that (95) has uncountably bounded positive solutions in $A(N, M)$. On the other hand, for any $c \in(N, M)$, there exist $\theta \in(0,1)$ and $T \geq$ $n_{0}+\tau_{0}+|\beta|$ such that the Mann iterative sequence with errors $\left\{x_{m}\right\}_{m \geq 0}$ generated by (56) converges to a bounded positive solution $x \in A(N, M)$ of (95) and has the error estimate (21), where $\left\{\gamma_{\eta}\right\}_{\eta \geq 0}$ is an arbitrary sequence in $A(N, M)$ and $\left\{\alpha_{\eta}\right\}_{\eta \geq 0}$ and $\left\{\beta_{\eta}\right\}_{\eta \geq 0}$ are any sequences in $[0,1]$ satisfying (22).

Example 6. Consider the second-order nonlinear neutral delay difference equation with forcing term

$$
\begin{aligned}
& \Delta\left((-1)^{n(n+1) / 2} n^{5}\left(\frac{x_{2^{n}+1}^{2}+1}{4 x_{n^{2}+n+1}^{2}+4 x_{n^{2}+n+1}+5}\right)\right. \\
& \left.\cdot \Delta\left(x_{n}-x_{n-\tau_{0}}\right)\right)+\frac{x_{n+2}+x_{4 n^{2}+n-1} \sin ^{2} n}{n^{4}+x_{n+2}^{4}+x_{4 n^{2}+n-1}^{2}} \\
& =\frac{(-1)^{n}+n}{n^{6}+4 n^{5}+1}, \quad n \geq 1,
\end{aligned}
$$

where $\tau_{0} \in \mathbb{N}$ is fixed. Let $n_{0}=1, m=k=2, M=5, N=2$, $\beta=1-\tau_{0}$,

$$
\begin{aligned}
& a_{n}=(-1)^{n(n+1) / 2} n^{5}, \\
& q_{n}=1, \\
& b_{n}=\frac{(-1)^{n}+n}{n^{6}+4 n^{5}+1}, \\
& \tau_{1 n}=-2^{n}+n-1, \\
& \tau_{2 n}=-n^{2}-1, \\
& \sigma_{1 n}=-2, \\
& \sigma_{2 n}=-4 n^{2}+1,
\end{aligned}
$$

$$
\begin{aligned}
f(n, u, v) & =\frac{u+v \sin ^{2} n}{n^{4}+u^{4}+v^{2}}, \\
h(u, v) & =\frac{v^{2}+1}{4 u^{2}+4 u+5}, \\
P_{n} & =\frac{2}{n^{4}}+\frac{4 M^{2}\left(1+2 M^{2}\right)}{n^{8}},
\end{aligned}
$$




$$
\begin{aligned}
& W_{n}=\frac{2 M}{n^{4}} \\
& L_{h}=\frac{4+18 M+4 M^{2}+24 M^{3}}{\left(4 N^{2}+4 N+5\right)^{2}}, \\
& m_{h}=\frac{1}{4 M^{2}+4 M+5},
\end{aligned}
$$$$
n \in \mathbb{N}_{n_{0}}, u, v \in \mathbb{R}
$$

It is easy to show that (15) (18) and (68) are satisfied. Note that

$$
\begin{aligned}
& \sum_{i=n_{0}+1}^{\infty} \frac{i}{\left|a_{i}\right|} \sum_{s=n_{0}}^{i-1} \max \left\{W_{s}, P_{s},\left|b_{s}\right|\right\}=\sum_{i=n_{0}+1}^{\infty} \frac{1}{i^{4}} \\
& \quad \cdot \sum_{s=n_{0}}^{i-1} \max \left\{\frac{2 M}{s^{4}}, \frac{2}{s^{4}}\right. \\
& \left.+\frac{4 M^{2}\left(1+2 M^{2}\right)}{s^{4}},\left|\frac{(-1)^{s}+s}{s^{6}+4 s^{5}+1}\right|\right\} \leq \sum_{i=n_{0}+1}^{\infty} \frac{1}{i^{4}} \\
& \quad \cdot \sum_{s=n_{0}}^{i-1} \frac{2+4 M^{2}\left(1+2 M^{2}\right)}{s^{4}} \leq 4\left(1+2 M^{2}+4 M^{4}\right) \\
& \cdot \sum_{i=n_{0}+1}^{\infty} \frac{1}{i^{4}}<+\infty
\end{aligned}
$$

that is, (69) holds. It follows from Theorem 8 that (98) has uncountably bounded positive solutions in $A(N, M)$. On the other hand, for any $b \in(N, M)$, there exist $\theta \in(0,1)$ and $T \geq$ $n_{0}+\tau_{0}+|\beta|$ such that the Mann iterative sequence with errors $\left\{x_{m}\right\}_{m \geq 0}$ generated by (70) converges to a bounded positive solution $x \in A(N, M)$ of (98) and has the error estimate (21), where $\left\{\gamma_{\eta}\right\}_{\eta \geq 0}$ is an arbitrary sequence in $A(N, M)$ and $\left\{\alpha_{\eta}\right\}_{\eta \geq 0}$ and $\left\{\beta_{\eta}\right\}_{\eta \geq 0}$ are any sequences in $[0,1]$ satisfying (22).

\section{Competing Interests}

The authors declare that there are no competing interests regarding the publication of this paper.

\section{Acknowledgments}

This study was supported by research funds from Dong-A University.

\section{References}

[1] R. P. Agarwal, S. R. Grace, and D. O’Regan, "Nonoscillatory solutions for discrete equations," Computers \& Mathematics with Applications, vol. 45, no. 6-9, pp. 1297-1302, 2003.

[2] R. P. Agarwal, M. M. S. Manuel, and E. Thandapani, "Oscillatory and nonoscillatory behavior of second order neutral delay difference equations," Mathematical and Computer Modelling, vol. 24, no. 1, pp. 5-11, 1996.
[3] J. Cheng, "Existence of a nonoscillatory solution of a secondorder linear neutral difference equation," Applied Mathematics Letters, vol. 20, no. 8, pp. 892-899, 2007.

[4] J. Cheng and Y. Chu, "Oscillation theorem for second-order difference equations," Taiwanese Journal of Mathematics, vol. 12, no. 3, pp. 623-633, 2008.

[5] H. A. El-Morshedy, "New oscillation criteria for second order linear difference equations with positive and negative coefficients," Computers \& Mathematics with Applications, vol. 58, no. 10, pp. 1988-1997, 2009.

[6] S. R. Grace and H. A. El-Morshedy, "Oscillation and nonoscillation theorems for certain second-order difference equations with forcing term," Journal of Mathematical Analysis and Applications, vol. 216, no. 2, pp. 614-625, 1997.

[7] J. Jiang and X. Li, "Oscillation criteria for second-order linear difference equations," Applied Mathematics and Computation, vol. 145, no. 2-3, pp. 591-601, 2003.

[8] X. Li and J. Jiang, "Oscillation of second-order linear difference equations," Mathematical and Computer Modelling, vol. 35, no. 9-10, pp. 983-990, 2002.

[9] Z. Liu, S. M. Kang, and J. S. Ume, "Existence of uncountably many bounded nonoscillatory solutions and their iterative approximations for second order nonlinear neutral delay difference equations," Applied Mathematics and Computation, vol. 213, no. 2, pp. 554-576, 2009.

[10] Z. Liu, H. Wu, S. M. Kang, and Y. C. Kwun, "On positive solutions and Mann iterative schemes of a third order difference equation," Abstract and Applied Analysis, vol. 2014, Article ID 470181, 16 pages, 2014.

[11] Z. Liu, X. P. Zhang, S. M. Kang, and Y. C. Kwun, "On positive solutions of a fourth order nonlinear neutral delay difference equation," Abstract and Applied Analysis, vol. 2014, Article ID 238036, 29 pages, 2014.

[12] Z. Liu, Y. Xu, and S. M. Kang, "Global solvability for a second order nonlinear neutral delay difference equation," Computers \& Mathematics with Applications, vol. 57, no. 4, pp. 587-595, 2009.

[13] X. Li and Y. Zhou, "Asymptotic behaviour and existence of nonoscillatory solutions of second-order neutral delay difference equations," Journal of Applied Mathematics \& Computing, vol. 11, no. 1-2, pp. 173-183, 2003.

[14] M. Ma, H. Tang, and W. Luo, "Periodic solutions for nonlinear second-order difference equations," Applied Mathematics and Computation, vol. 184, no. 2, pp. 685-694, 2007.

[15] L. Malaguti, P. Řehák, and V. Taddei, "Bounded solutions and wavefronts for discrete dynamics," Computers \& Mathematics with Applications, vol. 47, pp. 1079-1094, 2004.

[16] R. N. Rath, J. G. Dix, B. L. Barik, and B. Dihudi, "Necessary conditions for the solutions of second order non-linear neutral delay difference equations to be oscillatory or tend to zero," International Journal of Mathematics and Mathematical Sciences, vol. 2007, Article ID 60907, 16 pages, 2007.

[17] Y. G. Sun, "Oscillation and non-oscillation for second-order linear difference equations," Applied Mathematics and Computation, vol. 170, no. 2, pp. 1095-1103, 2005.

[18] Z. Szafrański and B. Szmanda, "Oscillation theorems for some nonlinear difference equations," Applied Mathematics and Computation, vol. 83, no. 1, pp. 43-52, 1997.

[19] E. Thandapani, R. Arul, and P. S. Raja, "The asymptotic behavior of nonoscillatory solutions of nonlinear neutral type difference equations," Mathematical and Computer Modelling, vol. 39, no. 13, pp. 1457-1465, 2004. 
[20] E. Thandapani, R. Arul, and P. S. Raja, "Bounded oscillation of second order unstable neutral type difference equations," Journal of Applied Mathematics \& Computing, vol. 16, no. 1-2, pp. 79-90, 2004.

[21] B. G. Zhang and Y. Zhou, "Oscillation and nonoscillation for second-order linear difference equations," Computers \& Mathematics with Applications, vol. 39, no. 1-2, pp. 1-7, 2000.

[22] L. S. Liu, "Ishikawa and Mann iterative process with errors for nonlinear strongly accretive mappings in Banach spaces," Journal of Mathematical Analysis and Applications, vol. 194, no. 1, pp. 114-125, 1995. 


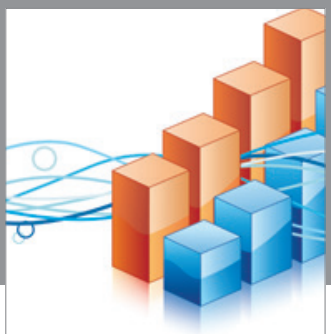

Advances in

Operations Research

vatem alat4

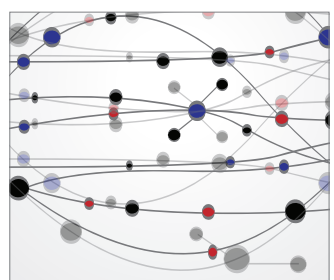

\section{The Scientific} World Journal
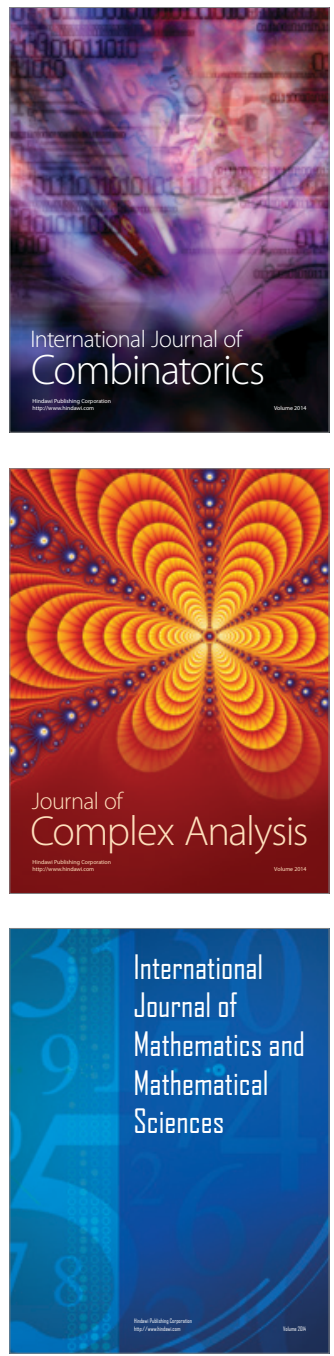
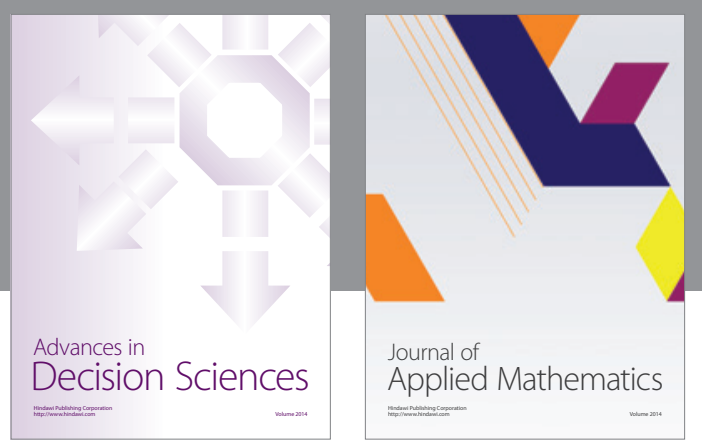

Algebra

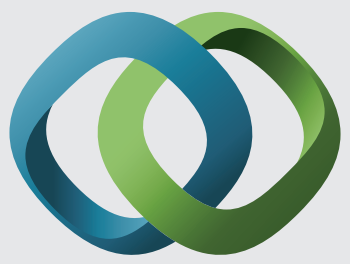

\section{Hindawi}

Submit your manuscripts at

http://www.hindawi.com
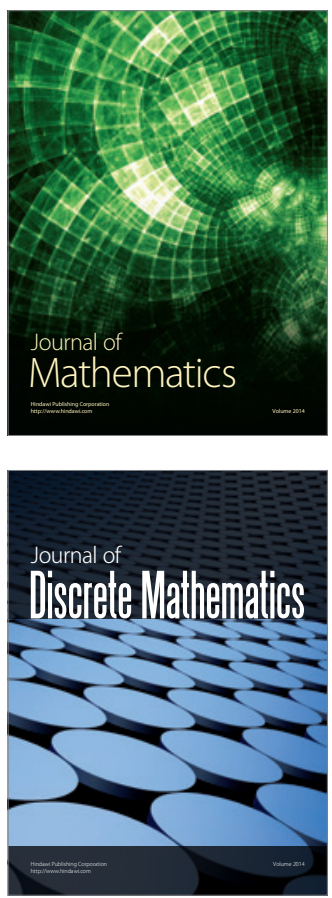

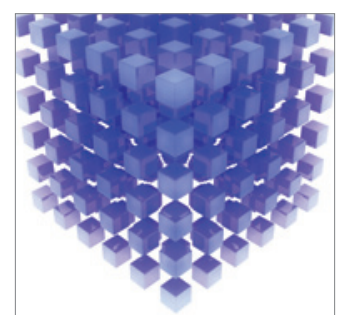

Mathematical Problems in Engineering
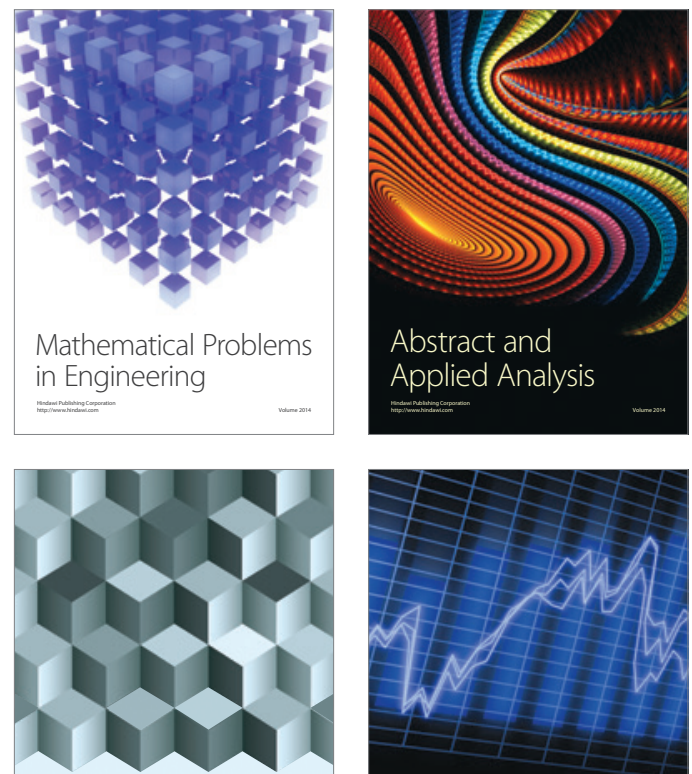

Journal of

Function Spaces

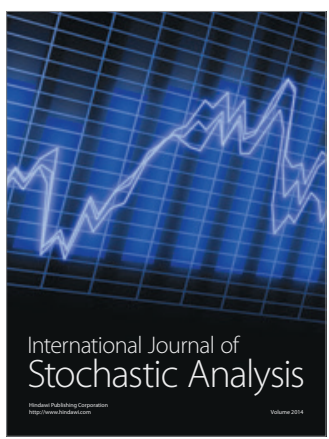

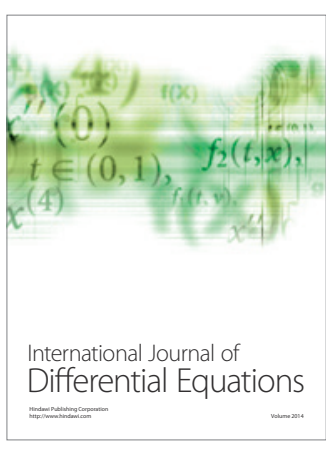
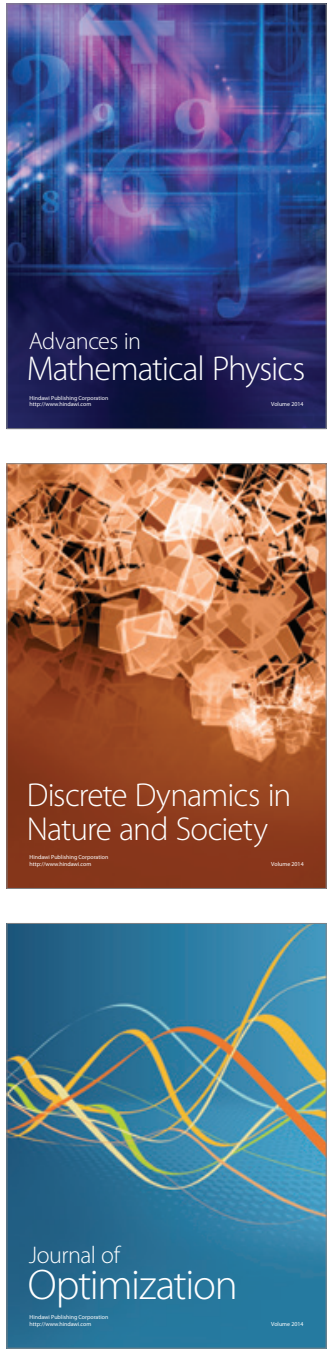